\title{
Melatonin alleviates hyperglycemic-induced cardiomyocyte apoptosis via regulation of long non- coding RNA H19/miR-29c/MAPK axis in diabetic cardiomyopathy
}

Haitao Tang

Anhui Medical University

Hongli Zhong

Hefei Affiliated Hospital of Anhui Medical University: Hefei Second People's Hospital

Wanqing Liu

Anhui Medical University

Tianfeng Hua

The Second Affiliated Hospital of Anhui Medical University

\section{Yi Wang}

Anhui Medical University

Zhenzhen Li

The First People's Hospital of Bengbu

\section{Yongxin Chen}

Anhui Medical University

\section{Zhangyong Dan}

Anhui Medical University

\section{Dongqing Wu}

Anhui Medical University

Qing Zhou

Anhui Medical University

\section{Yuan Wang}

Anhui Medical University

\section{Liuqing Wang}

The Third Clinical School of Hefei of Anhui Medical University

Hongxia Li

The Third Affiliated Hospital of Anhui Medical University

\section{Songtao Tang}

First Affiliated Hospital of Anhui Medical University

Huaqing Zhu ( $\nabla$ aydzhq@126.com )

Anhui Medical University https://orcid.org/0000-0002-0819-9232 
Original investigation

Keywords: melatonin, diabetic cardiomyopathy, apoptosis, non-coding RNA, MAPK

Posted Date: May 10th, 2021

DOl: https://doi.org/10.21203/rs.3.rs-449005/v1

License: (c) (1) This work is licensed under a Creative Commons Attribution 4.0 International License. Read Full License 


\section{Abstract}

\section{Background}

Recent studies revealed that non-coding RNAs (ncRNAs) play a crucial role in pathophysiological processes involving diabetic cardiomyopathy that contributes to heart failure. The present study was designed to further investigate the anti-apoptotic effect of melatonin on cardiomyocyte in diabetic condition and to elucidate the potential mechanisms associated with ncRNAs.

\section{Methods}

In vivo, langendorff-perfusion system and histology staining were used to assess the effect of melatonin on cardiac function. Reverse transcription-quantitative polymerase chain reaction (RT-qPCR) was used to measure the expression of ncRNAs. Protein expression levels were assessed by western bolt analysis. In vitro, hoechst 33258 staining and western bolt analysis were used to evaluate the effect of melatonin on apoptosis. We preformed luciferase assay and RNA immunoprecipitation to determine the targets of ncRNAs. RT-qPCR was used to observe the expression of ncRNAs in cardiomyocyte with high glucose treatment.

\section{Results}

In animal models, our results indicated that melatonin notably alleviated cardiac dysfunction and mitigated cardiomyocyte apoptosis in diabetic rats. Interestingly, IncRNA H19 level was increased along with concomitant decrease of miR-29c level in diabetic rats. However, we demonstrated that melatonin significantly downregulated $\mathrm{H} 19$ level and upregulated miR-29c level in vivo. In vitro experiments, it has been verified that positive modulation of miR-29c and inhibition of IncRNA H19 as well as mitogenactivated protein kinase (MAPK) pathways distinctly attenuated apoptosis in high glucose-treated H9c2 cells. Luciferase activity assay was conducted to evaluate the potential target sites of miR-29c on IncRNA $\mathrm{H} 19$ and MAPK13. LncRNA H19 silencing significantly downregulated the expression of the miR-29c target gene MAPK13 via inducing miR-29c expression. Furthermore, MAPK signal pathways were also affected through regulation of $\mathrm{H} 19$ and miR-29c. Most importantly, our results showed that melatonin alleviated hyperglycemic-induced cardiomyocyte apoptosis via inhibiting IncRNA H19/MAPK and increasing miR-29c level in vitro.

\section{Conclusions}

These results elucidate a novel protective mechanism of melatonin on diabetic cardiomyocyte apoptosis, which associated with the effect of melatonin on IncRNA H19/miR-29c expression and its downstream MAPK signal pathways, providing a promising strategy for preventing DCM in diabetic patients.

\section{Background}


Diabetes has become one of the most prevalent metabolic disorders in recent years. Cardiovascular complications are the leading cause of mortality and morbidity in diabetes. Diabetic cardiomyopathy (DCM), which develops in the absence of overt myocardial ischemia or hypertension, can trigger heart failure in patients and seriously endangers the lives of patients [1-3]. It has been reported that DCM can cause cardiac systolic and diastolic dysfunction and various metabolic disorders, which lead to the stress response of cardiomyocytes and death ultimately [4,5]. Actually, the precise pathogenesis of DCM is still unclear, while cardiomyocyte apoptosis is considered to be one of the major mechanisms. There are strong evidences showing that alleviation of cardiomyocyte apoptosis is effective for the prevention and treatment of DCM [6-10].

It has been proven that more than $90 \%$ of the genome is transcribed into non-coding RNAs by sequencing experiments. Long non-coding RNAs (IncRNAs) and micro RNAs(miRNAs) are two different kinds of noncoding RNAs. Interestingly, IncRNAs can act as sponges binding to miRNAs and affect the function of target miRNAs, which bind to the 3'-UTR of target genes to regulate their expressions at the posttranscriptional level. Non-coding RNAs(ncRNAs) are involved in the control of multiple cellular activities such as proliferation, differentiation and apoptosis [11-13]. Numerous studies have suggested that ncRNAs play a key role in regulating pathophysiological processes involved in DCM and aberrant expression of IncRNAs/miRNAs may have repercussions in cardiomyocyte activities $[14,15]$. For the past few years, it was extensively noticed that IncRNA H19 and miRNA-29c played a vital role in the development and progression of cardiovascular diseases. LncRNA H19 was up-regulated in the progression of atherosclerosis by regulating MAPK and NF-KB signaling pathway [16, 17]. Meanwhile, miRNA-29c decreased myocardial ischemia-reperfusion injury through inhibition of immoderate autophagy [18]. MicroRNA-29c also inhibited migration and angiogenesis of human endothelial cells by depressing insulin like growth factor 1 [19]. However, the related mechanisms of IncRNA H19/miR-29c involved in DCM are yet to be further investigated.

Melatonin (MLT, N-acetyl-5-methoxytryptamine), a pleiotropic molecule, is primarily secreted by the pineal gland, which is known as its anti-oxidant, anti-inflammatory and anti-apoptotic effects [8, 20-22]. Because of the function of scavenging free radicals directly, melatonin plays a critical role in alleviating many chronic diseases $[23,24]$. Evidences have been presented for the role of melatonin in the pathological process of cardiovascular diseases. Yu et al. found that melatonin attenuated DCM and reduced myocardial ischemia-reperfusion injury by improving mitochondrial quality control via SIRT6 [25]. Ding et al. elucidated that melatonin participated in preventing Drp1-mediated mitochondrial fission by regulating SIRT1-PGC1 a pathway [26]. In our previous research, melatonin not only prevented diabetic cardiomyocyte apoptosis via inhibiting endoplasmic reticulum stress, but also ameliorated diabetic arterial endothelial permeability by regulating MAPK signaling pathway activation [27, 28]. Intriguingly, previous studies have demonstrated the intimate relationship between cardiomyocyte apoptosis and MAPK signal pathways. Coincidentally, it was reported that inhibiting MAPK signaling could reduce cardiomyocyte apoptosis induced by high glucose in literatures. For example, it was certified that angiotensin-(1-7) protected cardiomyocytes against high glucose-induced injury and apoptosis through inhibiting ROS-activated leptin-p38 MAPK and ERK1/2 pathways [29]. Zhao et al. found that exogenous 
hydrogen sulfide ameliorated hyperglycemic-induced myocardial injury, inflammation and apoptosis via the CIRP-MAPK signaling pathway [30]. On the basis of above evidences, we hypothesized that melatonin could exert an ameliorative effect on cardiomyocyte apoptosis in DCM via blocking MAPK signal pathways.

Since ncRNAs open up a potential as biomarkers in DCM, the interaction between ncRNAs and MAPK signal pathways in cardiomyocyte remains an open question. Moreover, it has not been fully explored yet whether the beneficial effect of melatonin on cardiomyocyte apoptosis in DCM is mediated by regulation of ncRNAs networks. Thus, based on above hypothesis and questions, we developed DCM model in vivo and in vitro to broaden our understanding of the role of IncRNA H19/miRNA-29c/MAPK in the function of melatonin on cardiomyocyte apoptosis.

\section{Materials And Methods}

\section{Animals}

All animal experiments were conducted following the Guide for the Use and Care of Laboratory Animals. All experimental protocols were reviewed and approved by the Ethics Committee of Anhui Medical University (Hefei, China). Male Sprague Dawley (SD) rats (220-250 g, 8 weeks) were purchased from Beijing Vital River Laboratory Animal Technology Co., Ltd. (Beijing, China). The rats were acclimatized to the standard laboratory conditions for one week with normal water and chow available ad libitum after arrived. Afterwards, rats were randomized to normal, DM, DM + insulin and DM + MLT groups (12 rats in per group), and they were received a single injection of streptozotocin (STZ, $55 \mathrm{mg} / \mathrm{kg}$, Sigma-Aldrich; Merck KGaA, Darmstadt, Germany) except normal group. Animals were considered to be diabetic if the blood glucose levels tested higher than $11.1 \mathrm{mmol} / \mathrm{I}$ after 1 week. Diabetic rats were administered via injecting insulin ( $1 \mathrm{u} / \mathrm{kg} / \mathrm{d}$ within 10 weeks and $2 \mathrm{u} / \mathrm{kg} / \mathrm{d}$ after 10 weeks, Wanbang Biopharmaceuticals, China) and melatonin gavage (10 mg/kg/d, Nanjing Duly Biotech Co., Ltd., China) separately in DM + insulin and DM + MLT groups. During the experiment, all of them could consume the normative water and chow at liberty. At the end of week 16, the rats were anesthetized with a $10 \%$ chloral hydrate solution (300 $\mathrm{mg} / \mathrm{kg}$ ). With the heart fetched, cardiac function was immediately evaluated by the way of the Langendorff-perfusion system and then collected the myocardial tissues.

\section{Langendorff-perfusion system}

The Langendorff-perfusion system was used to record cardiac function in rats. Briefly, the heart was rapidly excised by thoracotomy under anesthesia, and the aorta was cannulated. The isolated heart was mounted on the Langendorff-perfusion system (Chengdu Techman Software Co., Ltd., China) and perfused with modified Krebs-Henseleit buffer aerated with $95 \% \mathrm{O}_{2}$ and $5 \% \mathrm{CO}_{2}$, yielding a final $\mathrm{pH} 7.4 \mathrm{in}$ $37^{\circ} \mathrm{C}$ circulating water bath. When stable, the parameters, which is related to cardiac function such as heart rate, were recorded via BL-420S biological function experiment system (Chengdu Techman Software Co., Ltd., China). 


\section{Histology}

The paraffin-embedded myocardial tissues were cut into 5- $\mu \mathrm{m}$ thick sections and stained with $\mathrm{H} \& \mathrm{E}$ (hematoxylin and eosin staining kit, Beyotime, China) or Masson's trichrome staining kit (Nanjing Jiancheng Technology Co., Ltd., China). The discrepant areas were captured by a DMI4000B fluorescence microscope (Leica, USA).

\section{Cell culture}

The cell line H9c2 obtained from the Cell Bank of Shanghai Institutes for Biological Sciences, Chinese Academy of Sciences were cultured in low-glucose Dulbecco's Modified Eagles Media (DMEM) (hyclone, USA) supplemented with $10 \%$ fetal bovine serum (FBS) (gbico, Australia) and $100 \mathrm{IU} / \mathrm{ml}$ penicillin/streptomycin (Beyotime, China) in $5 \% \mathrm{CO}_{2}$ at the temperature of $37^{\circ} \mathrm{C}$. The treatment of insulin $(1 \mu \mathrm{M})$ and melatonin $(10 \mu \mathrm{M})$ was treated $48 \mathrm{~h}$ in high glucose concentration.

\section{Cell transfection}

H19-shRNA (GenePharm Co., Ltd., Shanghai, China) was used to silence H19 expression. Simultaneously, miR-29c mimics and inhibitors (GenePharm) were used to regulate miR-29c expression. H9c2 cells were transfected with H19-shRNA, or miR-29c mimics, inhibitors, or controls (GenePharm) with Lipofectamine 2000 (Invitrogen; Thermo Fisher Scientific, Inc., Waltham, MA, USA) according to the manufacturer's protocol. A scrambled oligonucleotide (GenePharm) served as a control. Changes in RNA expression were determined by qRT-PCR $24 \mathrm{~h}$ after transfection, and differences in protein expression were measured via western blotting $48 \mathrm{~h}$ after transfection.

\section{Western blot analysis}

The proteins were separated by SDS-PAGE and transferred onto polyvinylidene difluoride (PVDF) membranes (Millipore, Massachusetts, USA) via electroblotting. The membranes were blocked in $5 \%$ skim milk for $2 \mathrm{~h}$ and incubated overnight at $4{ }^{\circ} \mathrm{C}$ with primary antibodies against $\mathrm{p}$ - JNK (Santa Cruz Biotechnology, USA, Cat\# sc-6254), JNK (Santa, Cat\# sc-7345), p-ERK (Santa, Cat\# sc-7383), ERK (Santa, Cat\# sc-135900), p-p38 (Santa, Cat\# sc-7975-R), p38 (Santa, Cat\# sc-7149), BAX (Santa, Cat\# sc-20067), Bcl-2 (Santa, Cat\# sc-7382), caspase-3 (Proteintech, Wuhan, China, Cat\# 11648-2-AP), caspase-9 (Cell Signaling Technology, USA, Cat\# 9508S) and GAPDH (Santa, Cat\# sc-32233). The proteins were detected with corresponding horseradish peroxidase-conjugated secondary antibodies coupled with ECL chemiluminescence detection kit (FDbio Science Biotech Co., Ltd., Hangzhou, China).

\section{Hoechst 33258 staining}

Cells were seeded in 24-well plates, cultured and treated, and the medium was thrown away. After washing with PBS 3 times, cells were fixed in fixative solution for $10 \mathrm{~min}$. The samples were incubated with Hoechst 33258 (Beyotime, China) staining solution for 5 min in darkness. Then, the plates were kept out light, observed and imaged using a fluorescence microscope. 


\section{Luciferase assay}

The 3'-UTR regions of IncRNA H19 and MAPK13, including potential miR-29c binding sites, were predicted with TargetScan version 7.11 and amplified by PCR. Subsequently, mutants were constructed by introducing point mutations into the seed binding site for miR-29c. The wild type and mutant fragments were subcloned into the firefly luciferase-expressing vector. H9c2 cells were seeded in 24-well plates and co-transfected with wild-type or mutated luciferase respectively. The Dual Luciferase Reporter Assay System (Promega) was used $48 \mathrm{~h}$ after transfection following the manufacturer's protocol. The relative luciferase activity was calculated according to the ratio of firefly luciferase activity to renilla luciferase activity.

\section{RNA immunoprecipitation (RIP)}

We investigated the direct interaction between miR-29c and IncRNA H19 by Argonaute 2 (Ago2)-RNA immunoprecipitation (Ago2-RIP). Anti-Ago2 (Sigma-Aldrich, USA), or control anti-IgG and Dynabeads Protein $\mathrm{G}$ (Invitrogen, USA) were pretreated at $4{ }^{\circ} \mathrm{C}$ with rotation $24 \mathrm{~h}$ in advance. Complete RIP lysis buffer, which contained protease inhibitor, phosphatase inhibitor and RNase inhibitor, was used to lyse cells. RNA in Ago2-RIP materials was washed several times with PEB buffer and treated with DNase I and Proteinase K. RNA was extracted with TRIzol (Invitrogen) and precipitated with absolute ethanol overnight at $-20^{\circ} \mathrm{C}$. After the removal of proteins and beads, RT-qPCR analysis of the purified RNA and IncRNA H19 enrichment in Ago2-RIP were put into effect.

\section{Reverse transcription-quantitative polymerase chain reaction (RT-qPCR)}

RNA from myocardial tissues and $\mathrm{H} 9 \mathrm{c} 2$ cells was isolated using TRIzol reagent and converted to complementary DNA (cDNA) using a First-Strand cDNA Synthesis Kit (Toyobo, Osaka, Japan). Then, the cDNA samples, added with Power SYBR green master mix (Applied Biosystems, Foster City, CA, USA), were subjected to qRT-PCR using a StepOne Real Time PCR system. The H19, miR-29c and mRNA level were respectively standardized by $\mathrm{U} 6$ and GAPDH. The amplification results were calculated on the basis of $2(-\triangle \Delta C t)$ method. The specific primers used were: H19 forward 5'-ATCGGTGCCTCAGCGTTCGG-3' and reverse 5'-CTGTCCTCGCCGTCACACCG-3'; MAPK13 forward 5'-GAGAAGGTGGCCATCAAGAA-3' and reverse 5'-GTCCTCATTCACAGCCAGGT-3; GAPDH forward 5'-GGTGGTCTCCTCTGACTTCAA-3' and reverse 5'-GTTGCTGTAGCCAAATTCGTTGT-3'; miR-29c, 5'-UAGCACCAUUUGAAAUCAGUGUU-3'; U6, 5'CGCTTCGGCAGCACATATACTAAAATTGGAAC-3'.

\section{Caspase-3 activity assay}

Caspase-3 activity was detected using a caspase-3 activity assay kit (Biomol Research Laboratories, Plymouth Meeting, PA) according to the manufacturer's protocol. After the cells lysed, total proteins were extracted and quantified using a protein assay kit. Subsequently, the proteins were incubated overnight at $37^{\circ} \mathrm{C}$ with acetyl-Asp-Glu-Val-Asp p-nitroanilide (Ac-DEVD-pNA) for the caspase-3 assay. The absorbance of pNA was detected by a microplate reader at $405 \mathrm{~nm}$. 


\section{DNA fragment assay}

DNA fragments were measured using a Cellular DNA Fragmentation ELISA kit (Roche Applied Science, Greenfield, IN, USA). Cells were seeded in 96-well plates. The medium was changed to serum-free medium after $24 \mathrm{~h}$, and continued to culture cells for an additional $24 \mathrm{~h}$. To label the DNA, the medium was replaced with 10\% FBS-DMEM supplemented with 5-bromo-2'-deoxyuridine. Following $24 \mathrm{~h}$ incubated, cells were treated with calcitriol for $4 \mathrm{~h}$ and MIS for $96 \mathrm{~h}$. Cells were lysed and then soluble DNA fragments were quantified by the Cellular DNA fragmentation ELISA kit according to the manufacturer's instructions.

\section{Statistical analysis}

Statistical analysis was performed using SPSS 20.0. The data were subjected to one-way ANOVA and parametric $t$-test and presented as means \pm standard deviation. All experiments were performed at least in triplicate. The results were considered to denote statistical significance if $P<0.05$.

\section{Results}

\section{Diabetes influenced body weight and blood glucose of rats.}

During the experiments, we measured the body weight and blood glucose once a week. The body weights of diabetic rats increased much slower than normal group (Fig. 1a), and blood glucose were at high levels all the time (Fig. 1b). Insulin treatment facilitated body weight gain and decreased blood glucose in diabetic rats. However, melatonin administration did not have these effects (Fig. 1a, b).

\section{Melatonin improved myocardial fiber disorder and collagen accumulation caused by diabetes.}

H\&E and Masson's trichrome staining showed that long-term diabetes impaired myocardial fiber (Fig. 2a) and aggravated collagen accumulation (Fig. 2b) significantly. Interestingly, the treatment of insulin and melatonin cured the diabetes-induced pathological injury (Fig. 2a, b).

\section{Melatonin improved myocardial dysfunction indiabetes.}

As is shown in the figures, heart rate, LVDP, coronary flow, $+\mathrm{dp} / \mathrm{dt}$ max and $-\mathrm{dp} / \mathrm{dt}$ max were distinctly decreased in DM group compared with normal group (Fig. 3a-e). These results indicated that long-term hyperglycemia contributed to myocardial dysfunction. Nevertheless, melatonin treatment improved cardiac function remarkably (Fig. 3a-e).

\section{Melatonin alleviated cardiomyocyte apoptosis caused by hyperglycemia.}

Western blot was applied to detect some proteins about apoptosis in diabetic rats and H9c2 in high glucose concentration. In vivo, the results showed that the expressions of cleaved-caspase3, cleavedcaspase9 and BAX/bcl-2 were obviously up-regulated in DM group compared with normal group, which indicated that hyperglycemia induced severe apoptosis. Surprisingly, the expressions of several proteins 
were normalizing with melatonin treatment (Fig. 4a-d). In vitro, the expressions of cleaved-caspase3, cleaved-caspase 9 and $\mathrm{BAX} / \mathrm{Bcl}-2$ were also increased remarkably in $\mathrm{H} 9 \mathrm{c} 2$ cells in high glucose concentration. Consistent with the results in vivo, the changes of apoptotic proteins expression were reversed via melatonin treatment (Fig. 4e-h). We also demonstrated that melatonin alleviated hyperglycemic-induced apoptosis in $\mathrm{H} 9 \mathrm{c} 2$ by hoechst 33258 staining (Fig. 4i).

\section{Melatonin suppressed phosphorylation of JNK/ERK/p38 in diabetic cardiomyocytes.}

In the DM group, the phosphorylation levels of JNK/ERK/p38 were significantly increased in myocardium compared with normal group. Whereas after insulin and melatonin treatment, the phosphorylation levels of JNK/ERK/p38 were inhibited strikingly (Fig. 5a-d). In H9c2 cells, the expressions of p-JNK, p-ERK and p-p38 were remarkably upregulated with stimulus of high glucose. While phosphorylation levels of above proteins fell down dramatically given the administrations of insulin and melatonin (Fig. 5e-h).

\section{Melatonin regulated the complex network of IncRNA H19, miRNA-29c and MAPK13 in cardiomyocytes.}

In diabetic rats, the expression of IncRNA H19 in myocardium was significantly downregulated after treatment with melatonin (Fig. 6a). Conversely, the expression of miRNA-29c in diabetic myocardium was dramatically upregulated by melatonin (Fig. 6b). In vitro, the expression of IncRNA H19 in H9c2 was significantly inhibited with H19-shRNA treatment, and IncRNA H19 expression was ulteriorly inhibited with both H19-shRNA and melatonin (Fig. 6c), indicating that melatonin further inhibited IncRNA H19 expression. On the contrary, the expression of miR-29c was obviously upregulated with both H19-shRNA and melatonin treatments (Fig. 6d). Intriguingly, MAPK13 protein expression was also significantly decreased after IncRNA H19 downregulation and melatonin further suppressed MAPK13 expression in H9c2 (Fig. 6e). With the miR-29c inhibitor treatment, the expression of miR-29c was distinctly suppressed, whereas it rose back with melatonin treatment simultaneously (Fig. 6f), indicating that melatonin promoted miR-29c expression. In contrast with H19-shRNA, miR-29c inhibitor increased MAPK13 protein expression in $\mathrm{H} 9 \mathrm{c} 2$, however, melatonin reversed the effect of miR-29c inhibitor (Fig. 6g). As expected, MAPK13 expression was affected in post-transcriptional level (Fig. 6h). Above results implied potential relationships among IncRNA H19/miR-29c, MAPK signal pathways and melatonin.

\section{LncRNA H19 binds miR-29c directly and MAPK13 is a target of miR-29c}

Luciferase assay result indicated that miR-29c mimic induced the decreases in wild type H19 luciferase activity. However, this effect was disappeared when a certain $\mathrm{H} 19$ site mutated, and there was no significant change between the miR-29c mimic group and control (Fig. 7a). Treatment with melatonin induced an analogous result like miR-29c mimic in H19 luciferase activity (Fig. 7b). RNA immunoprecipitation (RIP) experiment was performed to investigate whether $\mathrm{H} 19$ and miR-29c were components of the RNA-induced silencing complex. An Ago2 antibody was used to precipitate Ago2 protein from cultured cells (Fig. 7c). The mRNA expressions of both $\mathrm{H} 19$ and miR-29c were significantly gathered in the immunoprecipitates (Fig. 7d, e), suggesting that IncRNA H19 binds miR-29c directly. Meanwhile, another luciferase assay result showed that miRNA29c mimic caused inhibition in wild type 
MPAK13 luciferase activity. This effect was reduced after mutation at certain MAPK13 site (Fig. 7f). Similarly, the melatonin treatment decreased wild type MAPK13 rather than mutational MAPK13 luciferase activity (Fig. 7g).

\section{H19-shRNA, miRNA-29c mimic and melatonin treatments ameliorated hyperglycemic-induced apoptosis in $\mathrm{H} 9 \mathrm{c} 2$ cells}

Caspase-3 activity and DNA fragment assay were performed to determine the effects of H19-shRNA, miRNA-29c mimic and melatonin on cell apoptosis. High glucose could cause increased caspase-3 activity and DNA fragment in H9c2 cells (Fig. 8a, b). With transfection of H19-shRNA, caspase-3 activity was significantly downregulated in $\mathrm{H} 9 \mathrm{c} 2$ cells in high glucose condition (Fig. 8a). Similar with caspase-3 activity, the DNA fragment was also memorably downregulated in high glucose-treated cells with $\mathrm{H} 19$ shRNA transfection (Fig. 8b). Interestingly, we got consistent results with melatonin and miRNA-29c mimic treatments, which showed significant decreases in caspase-3 activity and DNA fragment (Fig. 8cf).

\section{Melatonin and H19-shRNA reversed the pro-apoptotic effect of miRNA-29c inhibitor in high glucose treated $\mathrm{H} 9 \mathrm{c} 2$ cells.}

Compared with normal glucose group, we detected significant increases of caspase-3 activity and DNA fragment in the high glucose group with miR-29c inhibitor transfection alone (Fig. 9a, b). We also observed remarkable inhibition of caspase-3 activity and DNA fragment with melatonin and H19-shRNA treatments respectively in high glucose groups (Fig. 9c-f). In brief, the pro-apoptotic effect of miR-29c inhibitor treatment was reversed by melatonin and H19-shRNA treatments in hyperglycemic condition.

\section{The underlying mechanism of melatonin is illustrated in schematic diagram.}

Schematic diagram illustrated that melatonin maintains ncRNAs homeostasis and reduces cardiomyocyte apoptosis in hyperglycemia condition via IncRNA H19/miR-29c/MAPK axis (Fig. 10).

\section{Discussion}

Currently, it lacks more effective medical therapies to halt the relentless progression of DCM. Thus, there is a necessary medical need to detect new therapeutic targets for treating DCM. In the present study, we verified that melatonin has an alleviative effect on hyperglycemic-induced cardiomyocyte apoptosis in vivo and vitro models. Our data for the first time unraveled that IncRNA H19/miR-29c/MAPK axis played a pivotal role in anti-apoptotic effect of melatonin in diabetic cardiomyopathy. These novel discoveries imply the possible therapeutic targets and propose potential clinical application of melatonin for DCM treatment.

The high risks of cardiovascular diseases have attracted much attention in diabetic patients [31, 32]. Long-term hyperglycemia is supposed to induce damage and cause subsequent apoptosis in cardiomyocytes [33, 34]. It has been reported that cardiomyocyte apoptosis exacerbated the development 
of DCM [35-37]. Our previous research found that persistent hyperglycemia aggravated endoplasmic reticulum stress response of cardiomyocytes, which in turn led to cell apoptosis [27]. It was reported that hyperglycemic-induced activation of JNK and p38 MAPK signaling pathways stimulated the expression of apoptosis-related proteins such as caspase 3, which in turn activated the apoptotic pathway and caused apoptosis of cardiomyocytes. However, when the activity of phosphorylation of JNK and p38 MAPK were suppressed, the cell apoptosis was also attenuated in diabetic cardiomyopathy models [3840]. In this study, we confirmed that MAPK signal pathways in cardiomyocytes were activated under hyperglycemic condition, which may elevate caspase-3 and 9 dependent apoptosis-related proteins expression and promote progression of apoptosis in diabetic myocardium.

Melatonin has been supposed as a potential therapeutic agent for DCM on account of its multiple physiological functions. Melatonin could take an antioxidative stress action via decreasing mTOR signaling pathway activation and rescue the impaired mitophagy activity through suppressing Mst1 in animals with DCM [41, 42]. It also published that melatonin could protect the rat heart against diabetesinduced apoptosis through ameliorating metabolic risk factors and modulating apoptotic proteins [43]. Our former research also revealed that melatonin played a positive role against the apoptosis via regulating endoplasmic reticulum stress and MAPK pathways $[27,28]$. In this study, our data showed that melatonin could not directly decrease blood glucose level in diabetic rats, but it could distinctly ameliorate diabetic cardiac dysfunction independent of regulation of blood glucose level, perhaps partly through reducing myocardial apoptosis via modulation of MAPK signal pathways and apoptosis-related proteins. We found that melatonin could improve cardiac dysfunction caused by hyperglycemia using Langendorff-perfusion System. Consistent with parameters of cardiac function, the results of morphologic staining further confirmed diabetes-induced myocardial tissue damage was cured with melatonin treatment. However, the underlying molecular mechanisms of melatonin in hyperglycemicinduced cardiomyocyte apoptosis are yet to be elucidated.

The competing endogenous RNAs (ceRNAs) networks, including IncRNAs and miRNAs interactions, were reportedly involved in the regulation of protein-coding genes implicated in the pathological process of DCM [14, 44-47]. LncRNA H19, one of the IncRNAs enriched in cardiovascular system, was reported to act as a ceRNA on different miRNAs to exert effects on cardiovascular complications $[48,49]$. Our previous study showed that IncRNA H19 suppression protected the endothelium against hyperglycemic-induced inflammation and oxidative stress via upregulating miR-29b expression and downregulating VEGFA expression, which caused activation of AKT/eNOS pathway in endothelial cells [11]. In this study, our results displayed that IncRNA H19 expression was negatively associated with miR-29c expression in myocardium of diabetic rats. Although it has been widely reported that melatonin alleviated diabetic cardiomyopathy at the protein level, the effects of melatonin on ceRNAs are rarely covered. Importantly, our study confirmed that melatonin partly inversed the increased IncRNA H19 and decreased miR-29c levels in diabetic cardiomyocytes. Moreover, our vitro experiments indicated that IncRNA H19 bound to miR-29c as a sponge and they interacted directly. Silence of IncRNA H19 further increased miR-29c expression in cardiomyocyte. We also observed that silence of IncRNA H19 suppressed the expression of MAPK13 protein in cardiomyocyte, which was testified in several studies. For example, IncRNA H19 can 
target at some RNAs to regulate MAPK signal pathways and promote the development of atherosclerosis and arterial calcification $[16,50]$. On the contrary, our data also showed that inhibition of miR-29c expression stimulated MAPK13 expression in cardiomyocyte and luciferase assay demonstrated that MAPK13 gene was the direct target of miR-29c. Thus, above results reveal that IncRNA H19 may in part regulate MAPK13 expression by competing with miR-29c. It is noteworthy that melatonin inhibits MAPK13 expression in cardiomyocyte via modulation of IncRNA19/miR-29c levels in our study. To investigate the role of IncRNA H19/miR-29c/MAPK13 signal in the regulation of cardiomyocyte apoptosis, H19 shRNA and miR-29c mimic were used to transfected with cardiomyocytes under hyperglycemia condition, which led to alleviation of apoptosis subsequently. In light of the role of MAPK signal pathways in apoptosis, we proved the potential involvement of IncRNA H19/miR-29c/MAPK13 signal pathway in the process of cardiomyocyte apoptosis in diabetes. Most importantly, our data showed that melatonin ameliorated cardiomyocyte apoptosis through modulation of IncRNA H19 mediated ceRNA network, which was confirmed for the first time.

\section{Conclusions}

In summary, we uncovered that melatonin treatment effectively ameliorated cardiac dysfunction and cardiomyocyte apoptosis in DCM, and the protection appeared to be largely dependent on the modulation of IncRNA H19/miR-29c/MAPK axis. These findings provide new insights that melatonin may be an attractive agent for alleviating the progression of DCM.

\section{Declarations}

\section{Ethics approval and consent to participate}

Animal experimental protocols were reviewed and approved by the Ethics Committee of Anhui Medical University.

\section{Consent for publication}

Not applicable.

\section{Availability of data and materials}

All data generated or analyzed during this study are included in this article.

\section{Competing interests}

The authors declare that they have no competing interests.

\section{Funding}


This study was supported by the National Natural Science Foundation of China (No 81570419 and No 81900750), Key Research and Development Project in Anhui Province (No 202004b11020025) and Special Support Program for Innovative and Entrepreneurial Talents of Anhui Province.

\section{Authors' contributions}

HTT, STT, HLZ, WQL, and TFH contributed to conduct the experiments and draft the manuscript. YW, ZZL, and $Y X C$ completed the data statistics of the experiments. ZYD and DQW completed the preliminary preparation of the experiments. QZ and YW critically revised the manuscript. HQZ, STT, LQW and HXL designed and supervised the research. All authors read and approved the final manuscript.

\section{Acknowledgements}

Not applicable.

\section{Author details}

${ }^{1}$ Laboratory of Molecular Biology, Department of Biochemistry, Anhui Medical University, Hefei, China. ${ }^{2}$ General Department of Hyperbaric Oxygen, Hefei Hospital Affiliated to Anhui Medical University, Hefei, China. ${ }^{3}$ Department of Intensive Care Unit, The Second Affiliated Hospital of Anhui Medical University, Hefei, China. ${ }^{4}$ Department of Clinical Laboratory, The First People's Hospital of Bengbu, Bengbu, China. ${ }^{5}$ Department of Clinical Laboratory, The Third Clinical School of Heifei of Anhui Medical University, Hefei, China. ${ }^{6}$ Department of Oncology, The Third Affiliated Hospital of Anhui Medical University, Hefei, China.

${ }^{7}$ Department of Endocrinology, The First Affiliated Hospital of Anhui Medical University, Hefei, China.

\section{Abbreviations}

ncRNAs: non-coding RNAs; RT-qPCR: Reverse Transcription-Quantitative Polymerase Chain Reaction; MAPK: mitogen-activated protein kinase; DCM: Diabetic cardiomyopathy; IncRNAs: long non-coding RNAs; miRNAs: microRNAs; MLT: melatonin; SD: Sprague Dawley; PVDF: polyvinylidene difluoride; cDNA: complementary DNA; RIP: RNA Immunoprecipitation; ceRNAs: competing endogenous RNAs.

\section{References}

1. Cole JB, Florez JC. Genetics of diabetes mellitus and diabetes complications. Nat Rev Nephrol. 2020 Jul;16(7):377-390.

2. Tan Y, Zhang Z, Zheng C, Wintergerst KA, Keller BB, Cai L. Mechanisms of diabetic cardiomyopathy and potential therapeutic strategies: preclinical and clinical evidence. Nat Rev Cardiol. 2020 Sep;17(9):585-607.

3. Murtaza G, Virk HUH, Khalid M, Lavie CJ, Ventura H, Mukherjee D, Ramu V, Bhogal S, Kumar G, Shanmugasundaram M, Paul TK. Diabetic cardiomyopathy - A comprehensive updated review. Prog 
Cardiovasc Dis. 2019 Jul-Aug;62(4):315-326.

4. Seferović PM, Paulus WJ. Clinical diabetic cardiomyopathy: a two-faced disease with restrictive and dilated phenotypes. Eur Heart J. 2015 Jul 14;36(27):1718-27, 1727a-1727c.

5. Shah SJ, Kitzman DW, Borlaug BA, van Heerebeek L, Zile MR, Kass DA, Paulus WJ. PhenotypeSpecific Treatment of Heart Failure With Preserved Ejection Fraction: A Multiorgan Roadmap. Circulation. 2016 Jul 5;134(1):73-90.

6. Yao Q, Ke ZQ, Guo S, Yang XS, Zhang FX, Liu XF, Chen X, Chen HG, Ke HY, Liu C. Curcumin protects against diabetic cardiomyopathy by promoting autophagy and alleviating apoptosis. J Mol Cell Cardiol. 2018 Nov;124:26-34.

7. Wang X, Pan J, Liu D, Zhang M, Li X, Tian J, Liu M, Jin T, An F. Nicorandil alleviates apoptosis in diabetic cardiomyopathy through PI3K/Akt pathway. J Cell Mol Med. 2019 Aug;23(8):5349-5359.

8. Zhang M, Lin J, Wang S, Cheng Z, Hu J, Wang T, Man W, Yin T, Guo W, Gao E, Reiter RJ, Wang H, Sun D. Melatonin protects against diabetic cardiomyopathy through Mst1/Sirt3 signaling. J Pineal Res. 2017 Sep;63(2).

9. Malek V, Gaikwad AB. Telmisartan and thiorphan combination treatment attenuates fibrosis and apoptosis in preventing diabetic cardiomyopathy. Cardiovasc Res. 2019 Feb 1;115(2):373-384.

10. Yin Z, Zhao Y, He M, Li H, Fan J, Nie X, Yan M, Chen C, Wang DW. MiR-30c/PGC-1 $\beta$ protects against diabetic cardiomyopathy via PPARa. Cardiovasc Diabetol. 2019 Jan 11;18(1):7.

11. Cheng XW, Chen ZF, Wan YF, Zhou Q, Wang H, Zhu HQ. Long Non-coding RNA H19 Suppression Protects the Endothelium Against Hyperglycemic-Induced Inflammation via Inhibiting Expression of miR-29b Target Gene Vascular Endothelial Growth Factor a Through Activation of the Protein Kinase B/Endothelial Nitric Oxide Synthase Pathway. Front Cell Dev Biol. 2019 Nov 1;7:263.

12. Yang S, Tang W, He Y, Wen L, Sun B, Li S. Long non-coding RNA and microRNA-675/let-7a mediates the protective effect of melatonin against early brain injury after subarachnoid hemorrhage via targeting TP53 and neural growth factor. Cell Death Dis. 2018 Jan 24;9(2):99.

13. Rupaimoole R, Slack FJ. MicroRNA therapeutics: towards a new era for the management of cancer and other diseases. Nat Rev Drug Discov. 2017 Mar;16(3):203-222.

14. Zhou X, Zhang W, Jin M, Chen J, Xu W, Kong X. IncRNA MIAT functions as a competing endogenous RNA to upregulate DAPK2 by sponging miR-22-3p in diabetic cardiomyopathy. Cell Death Dis. 2017 Jul 13;8(7):e2929.

15. Wang C, Liu G, Yang H, Guo S, Wang H, Dong Z, Li X, Bai Y, Cheng Y. MALAT1-mediated recruitment of the histone methyltransferase EZH2 to the microRNA-22 promoter leads to cardiomyocyte apoptosis in diabetic cardiomyopathy. Sci Total Environ. 2021 Apr 20;766:142191.

16. Pan JX. LncRNA H19 promotes atherosclerosis by regulating MAPK and NF-kB signaling pathway. Eur Rev Med Pharmacol Sci. 2017 Jan;21(2):322-328.

17. Bitarafan S, Yari M, Broumand MA, Ghaderian SMH, Rahimi M, Mirfakhraie R, Azizi F, Omrani MD. Association of Increased Levels of IncRNA H19 in PBMCs with Risk of Coronary Artery Disease. Cell J. 2019 Jan;20(4):564-568. 
18. Li T, Gu J, Yang O, Wang J, Wang Y, Kong J. Bone Marrow Mesenchymal Stem Cell-Derived Exosomal miRNA-29c Decreases Cardiac Ischemia/Reperfusion Injury Through Inhibition of Excessive Autophagy via the PTEN/Akt/mTOR Signaling Pathway. Circ J. 2020 Jul 22;84(8):1304-1311.

19. Hu Y, Deng F, Song J, Lin J, Li X, Tang Y, J, Tang T, Zheng L. Evaluation of miR-29c inhibits endotheliocyte migration and angiogenesis of human endothelial cells by suppressing the insulin like growth factor 1. Am J Transl Res. 2015 May 15;7(5):866-77.

20. Reiter RJ, Mayo JC, Tan DX, Sainz RM, Alatorre-Jimenez M, Qin L. Melatonin as an antioxidant: under promises but over delivers. J Pineal Res. 2016 Oct;61(3):253-78.

21. Gonzaga NA, Awata WMC, Ficher SP, Assis VO, Alves JV, Tostes RC, Tirapelli CR. Melatonin reverses the loss of the anticontractile effect of perivascular adipose tissue in obese rats. J Pineal Res. 2021 Mar;70(2):e12710.

22. Tan DX, Reiter RJ. An evolutionary view of melatonin synthesis and metabolism related to its biological functions in plants. J Exp Bot. 2020 Aug 6;71(16):4677-4689.

23. Jilg A, Bechstein P, Saade A, Dick M, Li TX, Tosini G, Rami A, Zemmar A, Stehle JH. Melatonin modulates daytime-dependent synaptic plasticity and learning efficiency. J Pineal Res. 2019 Apr;66(3):e12553.

24. Yin J, Li Y, Han H, Ma J, Liu G, Wu X, Huang X, Fang R, Baba K, Bin P, Zhu G, Ren W, Tan B, Tosini G, He X, Li T, Yin Y. Administration of Exogenous Melatonin Improves the Diurnal Rhythms of the Gut Microbiota in Mice Fed a High-Fat Diet. mSystems. 2020 May 19;5(3):e00002-20.

25. Yu LM, Dong X, Xue XD, Xu S, Zhang X, Xu YL, Wang ZS, Wang Y, Gao H, Liang YX, Yang Y, Wang HS. Melatonin attenuates diabetic cardiomyopathy and reduces myocardial vulnerability to ischemiareperfusion injury by improving mitochondrial quality control: Role of SIRT6. J Pineal Res. 2021 Jan;70(1):e12698.

26. Ding M, Feng N, Tang D, Feng J, Li Z, Jia M, Liu Z, Gu X, Wang Y, Fu F, Pei J. Melatonin prevents Drp1mediated mitochondrial fission in diabetic hearts through SIRT1-PGC1a pathway. J Pineal Res. 2018 Sep;65(2):e12491.

27. Xiong FY, Tang ST, Su H, Tang HQ, Jiang P, Zhou Q, Wang Y, Zhu HQ. Melatonin ameliorates myocardial apoptosis by suppressing endoplasmic reticulum stress in rats with long-term diabetic cardiomyopathy. Mol Med Rep. 2018 Jan;17(1):374-381.

28. Tang ST, Su H, Zhang Q, Tang HQ, Wang CJ, Zhou Q, Wei W, Zhu HQ, Wang Y. Melatonin Attenuates Aortic Endothelial Permeability and Arteriosclerosis in Streptozotocin-Induced Diabetic Rats: Possible Role of MLCK- and MLCP-Dependent MLC Phosphorylation. J Cardiovasc Pharmacol Ther. 2016 Jan;21(1):82-92.

29. Lei Y, Xu Q, Zeng B, Zhang W, Zhen Y, Zhai Y, Cheng F, Mei W, Zheng D, Feng J, Lan J, Chen J. Angiotensin-(1-7) protects cardiomyocytes against high glucose-induced injuries through inhibiting reactive oxygen species-activated leptin-p38 mitogen-activated protein kinase/extracellular signalregulated protein kinase $1 / 2$ pathways, but not the leptin-c-Jun $\mathrm{N}$-terminal kinase pathway in vitro. $\mathrm{J}$ Diabetes Investig. 2017 Jul;8(4):434-445. 
30. Zhao HL, Wu BQ, Luo Y, Zhang WY, Hao YL, Liang JJ, Fang F, Liu W, Chen XH. Exogenous hydrogen sulfide ameliorates high glucose-induced myocardial injury \& inflammation via the CIRP-MAPK signaling pathway in H9c2 cardiac cells. Life Sci. 2018 Sep 1;208:315-324.

31. Cai X, Zhang Y, Li M, Wu JH, Mai L, Li J, Yang Y, Hu Y, Huang Y. Association between prediabetes and risk of all cause mortality and cardiovascular disease: updated meta-analysis. BMJ. $2020 \mathrm{Jul}$ 15;370:m2297.

32. Kenny HC, Abel ED. Heart Failure in Type 2 Diabetes Mellitus. Circ Res. 2019 Jan 4;124(1):121-141.

33. Ren BC, Zhang YF, Liu SS, Cheng XJ, Yang X, Cui XG, Zhao XR, Zhao H, Hao MF, Li MD, Tie YY, Qu L, Li XY. Curcumin alleviates oxidative stress and inhibits apoptosis in diabetic cardiomyopathy via Sirt1-Foxo1 and PI3K-Akt signalling pathways. J Cell Mol Med. 2020 Nov;24(21):12355-12367.

34. Xu D, Zhang X, Chen X, Yang S, Chen H. Inhibition of miR-223 attenuates the NLRP3 inflammasome activation, fibrosis, and apoptosis in diabetic cardiomyopathy. Life Sci. 2020 Sep 1;256:117980.

35. Qi B, He L, Zhao Y, Zhang L, He Y, Li J, Li C, Zhang B, Huang Q, Xing J, Li F, Li Y, Ji L. Akap1 deficiency exacerbates diabetic cardiomyopathy in mice by NDUFS1-mediated mitochondrial dysfunction and apoptosis. Diabetologia. 2020 May;63(5):1072-1087.

36. Chen H, Tran D, Yang HC, Nylander S, Birnbaum Y, Ye Y. Dapagliflozin and Ticagrelor Have Additive Effects on the Attenuation of the Activation of the NLRP3 Inflammasome and the Progression of Diabetic Cardiomyopathy: an AMPK-mTOR Interplay. Cardiovasc Drugs Ther. 2020 Aug;34(4):443461.

37. Ge Q, Zhao L, Ren XM, Ye P, Hu ZY. LCZ696, an angiotensin receptor-neprilysin inhibitor, ameliorates diabetic cardiomyopathy by inhibiting inflammation, oxidative stress and apoptosis. Exp Biol Med (Maywood). 2019 Sep;244(12):1028-1039.

38. Al-Damry NT, Attia HA, Al-Rasheed NM, Al-Rasheed NM, Mohamad RA, Al-Amin MA, Dizmiri N, Atteya M. Sitagliptin attenuates myocardial apoptosis via activating LKB-1/AMPK/Akt pathway and suppressing the activity of GSK-3 $\beta$ and $\mathrm{p} 38 \mathrm{a} / \mathrm{MAPK}$ in a rat model of diabetic cardiomyopathy. Biomed Pharmacother. 2018 Nov; 107:347-358.

39. Zuo G, Ren X, Qian X, Ye P, Luo J, Gao X, Zhang J, Chen S. Inhibition of JNK and p38 MAPK-mediated inflammation and apoptosis by ivabradine improves cardiac function in streptozotocin-induced diabetic cardiomyopathy. J Cell Physiol. 2019 Feb;234(2):1925-1936.

40. Xiong Z, Li Y, Zhao Z, Zhang Y, Man W, Lin J, Dong Y, Liu L, Wang B, Wang H, Guo B, Li C, Li F, Wang $H$, Sun D. Mst1 knockdown alleviates cardiac lipotoxicity and inhibits the development of diabetic cardiomyopathy in db/db mice. Biochim Biophys Acta Mol Basis Dis. 2020 Aug 1;1866(8):165806.

41. Che H, Wang Y, Li H, Li Y, Sahil A, Lv J, Liu Y, Yang Z, Dong R, Xue H, Wang L. Melatonin alleviates cardiac fibrosis via inhibiting IncRNA MALAT1/miR-141-mediated NLRP3 inflammasome and TGFB1/Smads signaling in diabetic cardiomyopathy. FASEB J. 2020 Apr;34(4):5282-5298.

42. Kandemir YB, Tosun V, Güntekin Ü. Melatonin protects against streptozotocin-induced diabetic cardiomyopathy through the mammalian target of rapamycin (mTOR) signaling pathway. Adv Clin Exp Med. 2019 Sep;28(9):1171-1177. 
43. Wang S, Zhao Z, Feng X, Cheng Z, Xiong Z, Wang T, Lin J, Zhang M, Hu J, Fan Y, Reiter RJ, Wang H, Sun D. Melatonin activates Parkin translocation and rescues the impaired mitophagy activity of diabetic cardiomyopathy through Mst1 inhibition. J Cell Mol Med. 2018 Oct;22(10):5132-5144.

44. Amin AH, El-Missiry MA, Othman Al. Melatonin ameliorates metabolic risk factors, modulates apoptotic proteins, and protects the rat heart against diabetes-induced apoptosis. Eur $\mathrm{J}$ Pharmacol. 2015 Jan 15;747:166-73.

45. Sun H, Wang C, Zhou Y, Cheng X. Long noncoding RNA OIP5-AS1 overexpression promotes viability and inhibits high glucose-induced oxidative stress of cardiomyocytes by targeting microRNA34a/SIRT1 axis in diabetic cardiomyopathy. Endocr Metab Immune Disord Drug Targets. 2020 Dec 29.

46. Feng Y, Xu W, Zhang W, Wang W, Liu T, Zhou X. LncRNA DCRF regulates cardiomyocyte autophagy by targeting miR-551b-5p in diabetic cardiomyopathy. Theranostics. 2019 Jun 10;9(15):4558-4566.

47. Yang $F$, Qin $Y$, Lv J, Wang $Y$, Che $H$, Chen $X$, Jiang $Y$, Li A, Sun X, Yue E, Ren L, Li Y, Bai Y, Wang L. Silencing long non-coding RNA Kcnq1ot1 alleviates pyroptosis and fibrosis in diabetic cardiomyopathy. Cell Death Dis. 2018 Sep 24;9(10):1000.

48. Shi S, Song L, Yu H, Feng S, He J, Liu Y, He Y. Knockdown of LncRNA-H19 Ameliorates Kidney Fibrosis in Diabetic Mice by Suppressing miR-29a-Mediated EndMT. Front Pharmacol. 2020 Nov 26;11:586895.

49. Fawzy MS, Abdelghany AA, Toraih EA, Mohamed AM. Circulating long noncoding RNAs H19 and GAS5 are associated with type 2 diabetes but not with diabetic retinopathy: A preliminary study. Bosn J Basic Med Sci. 2020 Aug 3;20(3):365-371.

50. Liu F, Yang XC, Chen ML, Zhuang ZW, Jiang Y, Wang J, Zhou YJ. LncRNA H19/Runx2 axis promotes VSMCs transition via MAPK pathway. Am J TransI Res. 2020 Apr 15;12(4):1338-1347.

\section{Figures}

a

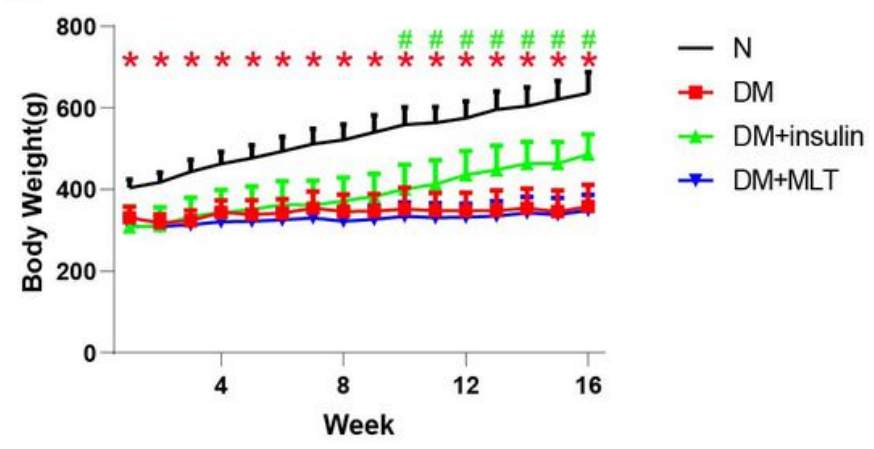

b

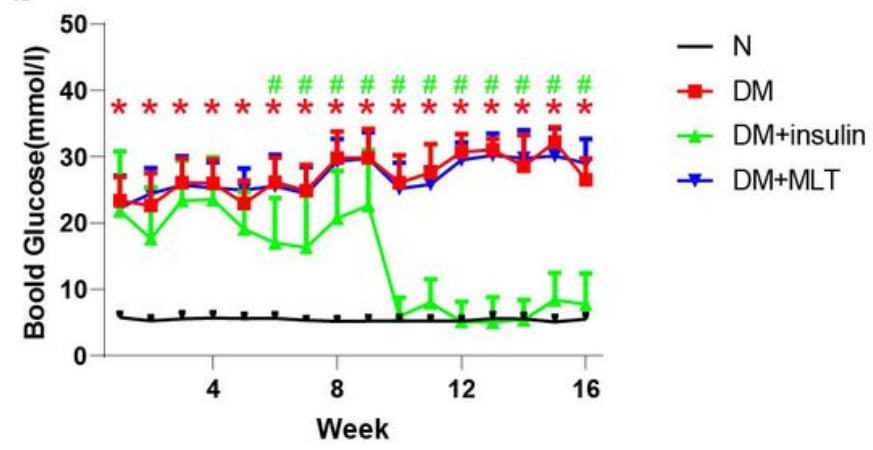

\section{Figure 1}


The body weight (a) and blood glucose (b) parameters of rats in different experimental groups $(\mathrm{n}=12$ per group). The parameters were measured by electronic scale and glucometer once a week. The data are presented as means \pm standard deviation. ${ }^{*} P<0.05$, DM group vs. normal group; $\# P<0.05, D M+$ insulin group vs. DM group.

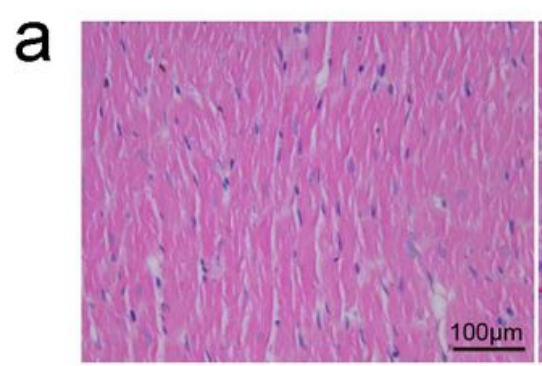

$\mathrm{N}$

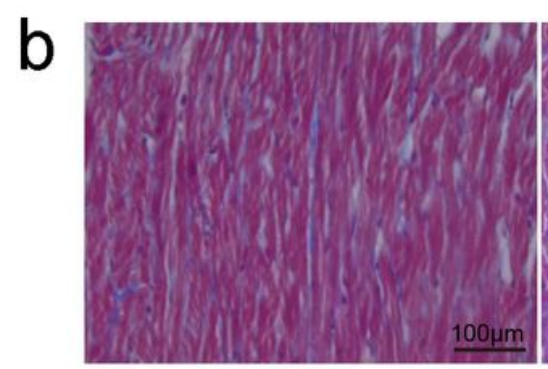

$\mathrm{N}$

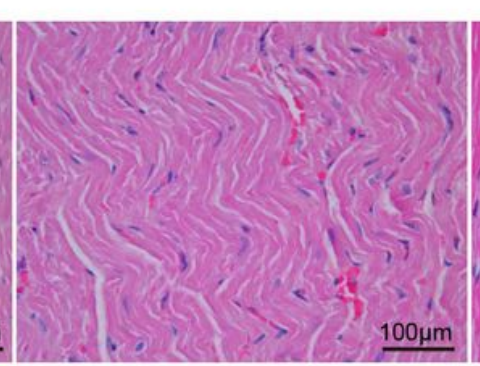

$\mathrm{DM}$

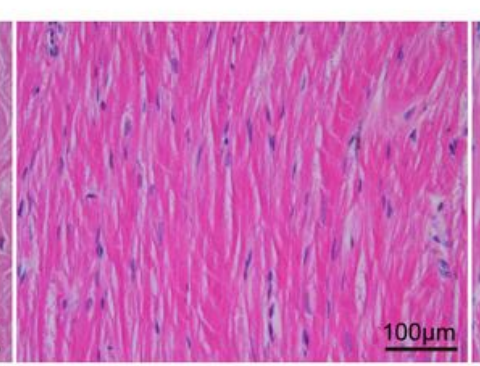

DM+insulin

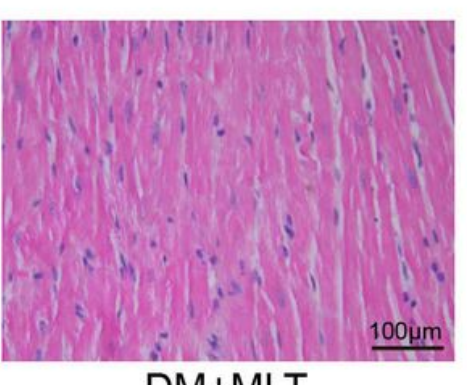

$\mathrm{DM}+\mathrm{MLT}$

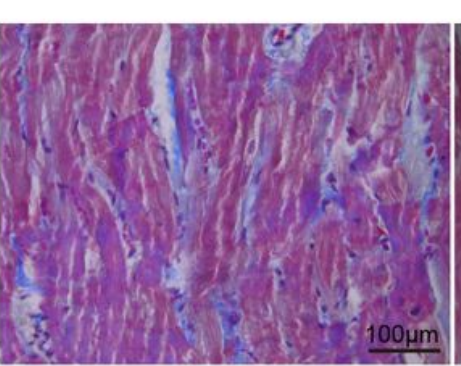

DM

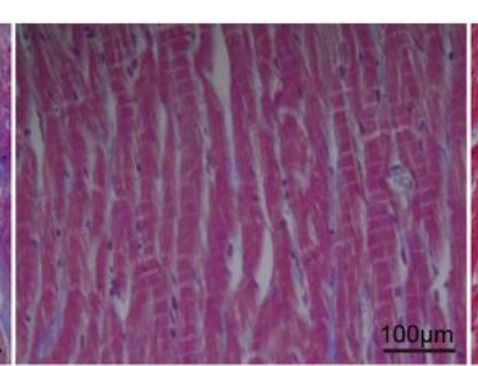

DM+insulin

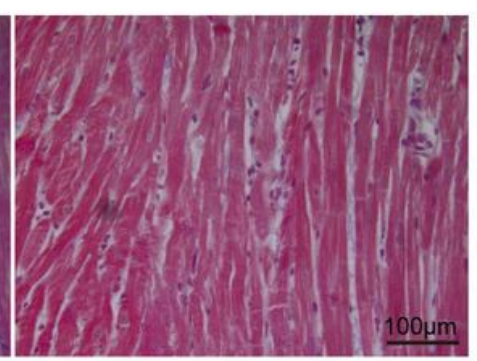

$\mathrm{DM}+\mathrm{MLT}$

\section{Figure 2}

Therapeutic effects of melatonin on histomorphology and fibrosis in myocardial tissues of diabetic rats. (a)H\&E staining showed that melatonin improved the cardiac fiber disorder in diabetes. (b)Masson's trichrome staining showed that melatonin alleviated cardiac collagen accumulation caused by diabetes. All experiments were performed at least in triplicate.
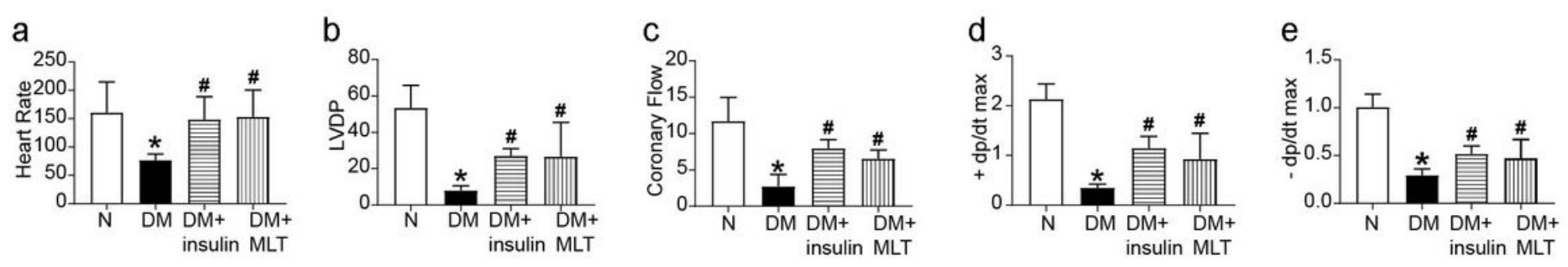

\section{Figure 3}

Melatonin relieved myocardial dysfunction in diabetic rats. The Langendorff-perfusion system was used to record myocardial dysfunction in rats ( $n=6$ per group). (a) heart rate; (b) left ventricular development pressure, LVDP; (c) coronary flow; (d) $+\mathrm{dp} / \mathrm{dt}$ max; (e) -dp/dt max. The data are presented as means \pm standard deviation. ${ }^{*} \mathrm{P}<0.05$ vs. normal group; $\# \mathrm{P}<0.05$ vs. DM group. 

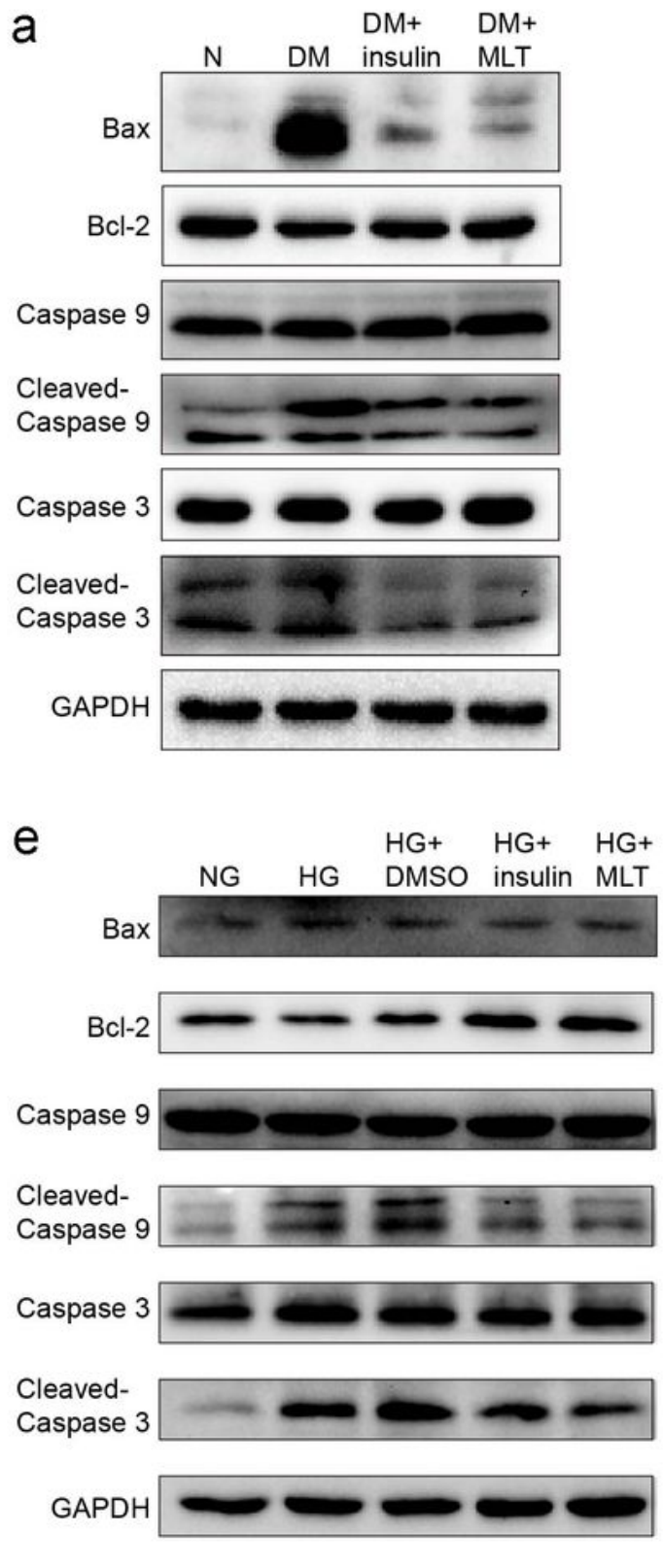
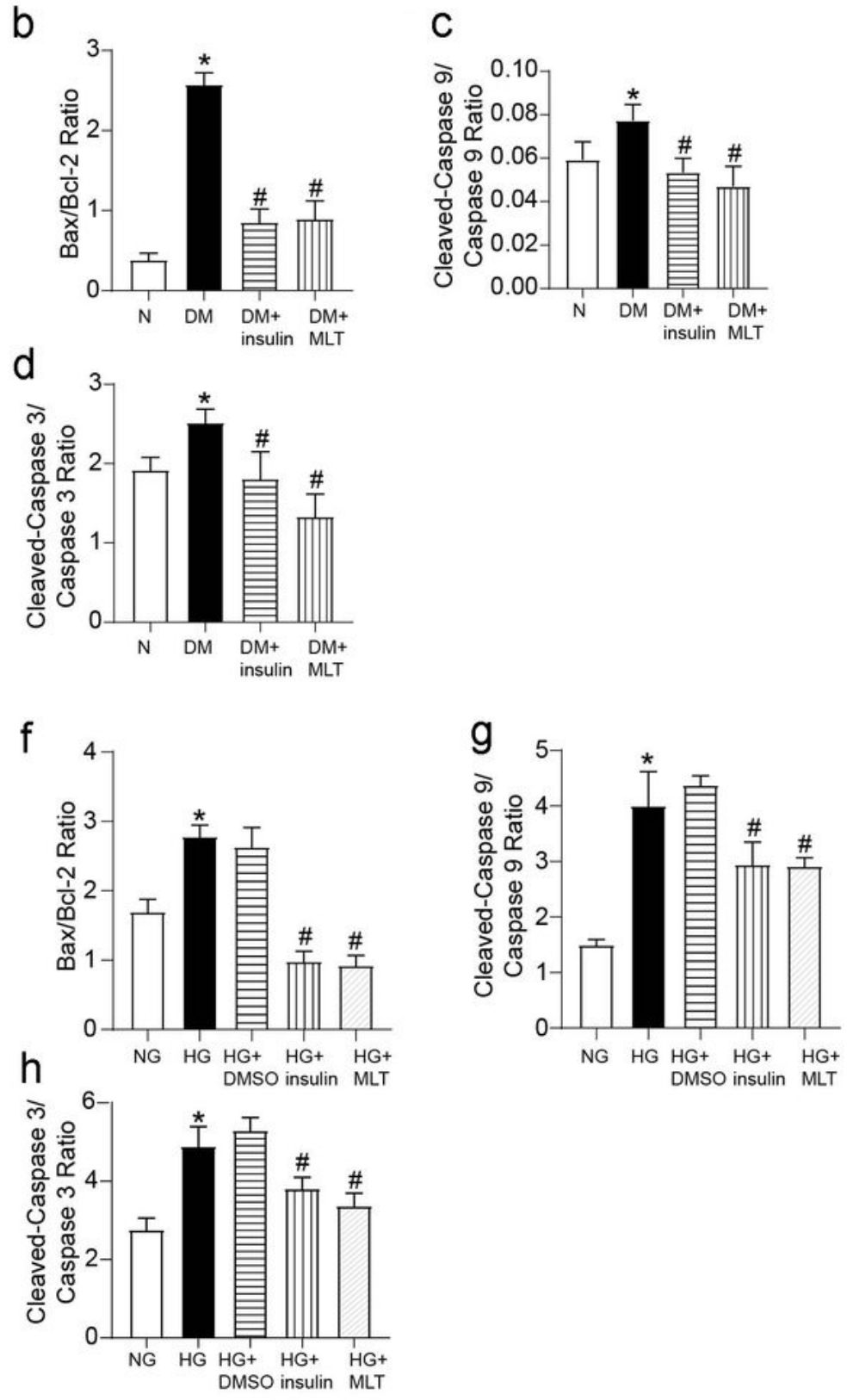

i NG

HG

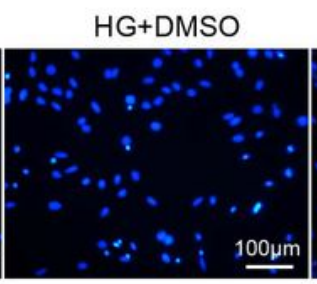

HG+insulin

HG+MLT
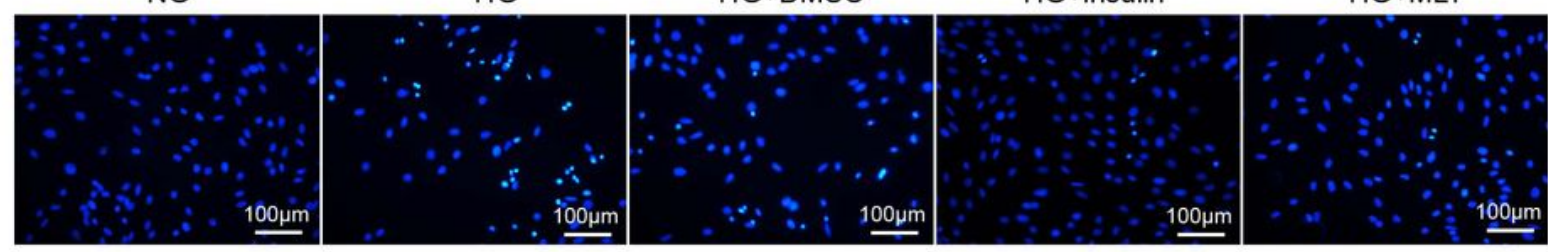

\section{Figure 4}

Melatonin alleviated apoptosis of cardiomyocyte in vitro and vivo. (a-d) Western blotting was used to detect the expression levels of cleaved-caspase3, cleaved-caspase 9 and BAX/Bcl-2 in myocardium of different groups in vivo. (e-h) Western blot analysis of the expression levels of cleaved-caspase3, cleavedcaspase9 and BAX/Bcl-2 in vitro experiment. (i) Hoechst 33258 staining showed that apoptosis of cardiomyocyte was relieved by melatonin treatment. The data are presented as means \pm standard 
deviation. All experiments were performed at least in triplicate. ${ }^{*} \mathrm{P}<0.05 \mathrm{vs}$. normal or normal glucose (NG: $5.5 \mathrm{mM}$ ) group; \#P $<0.05$ vs. DM or HG (HG: 33 mM) group.
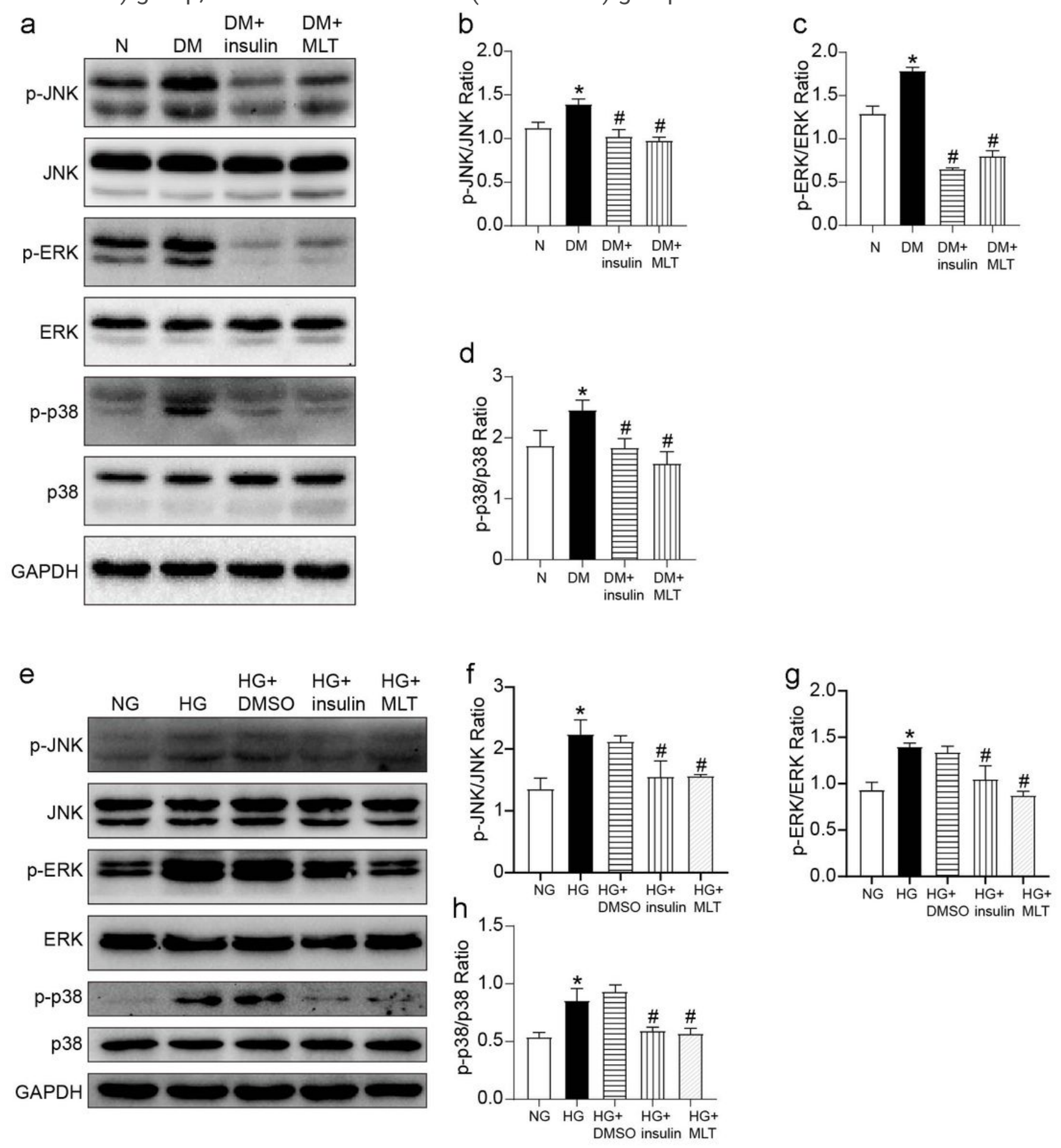

\section{Figure 5}

Melatonin reduced phosphorylated levels of JNK/ERK/p38 in diabetic myocardium and H9c2 cells. (a-d) The phosphorylated levels of JNK/ERK/p38 in myocardium were showed in different groups in vivo. (e-h) The phosphorylated levels of JNK/ERK/p38 in cardiomyocytes were showed in different groups in vitro. 
The data are presented as means \pm standard deviation. All experiments were performed at least in triplicate. *P < 0.05 vs. normal or NG (NG: 5.5 mM) group; \#P < 0.05 vs. DM or HG (HG: 33 mM) group.
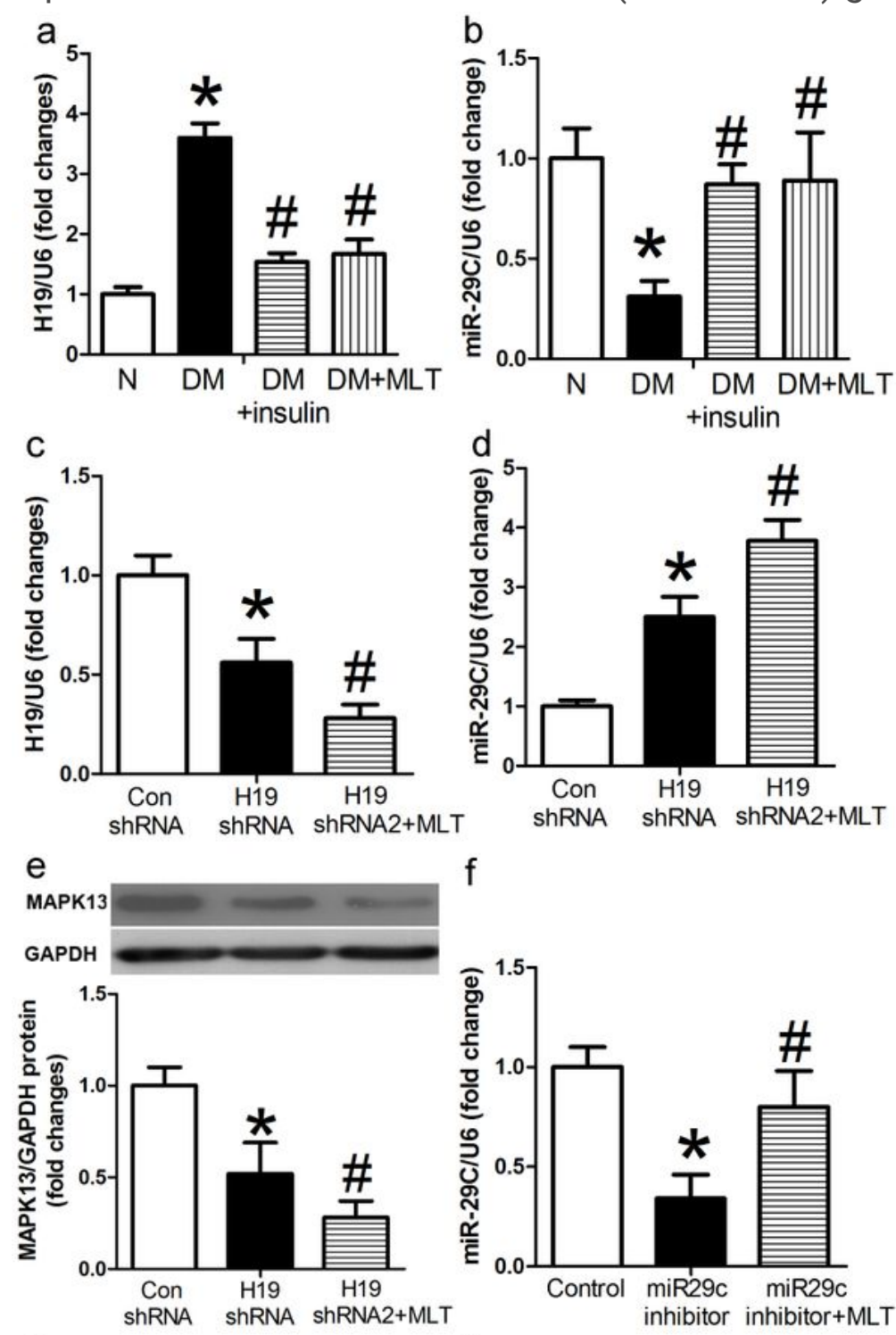

$f$
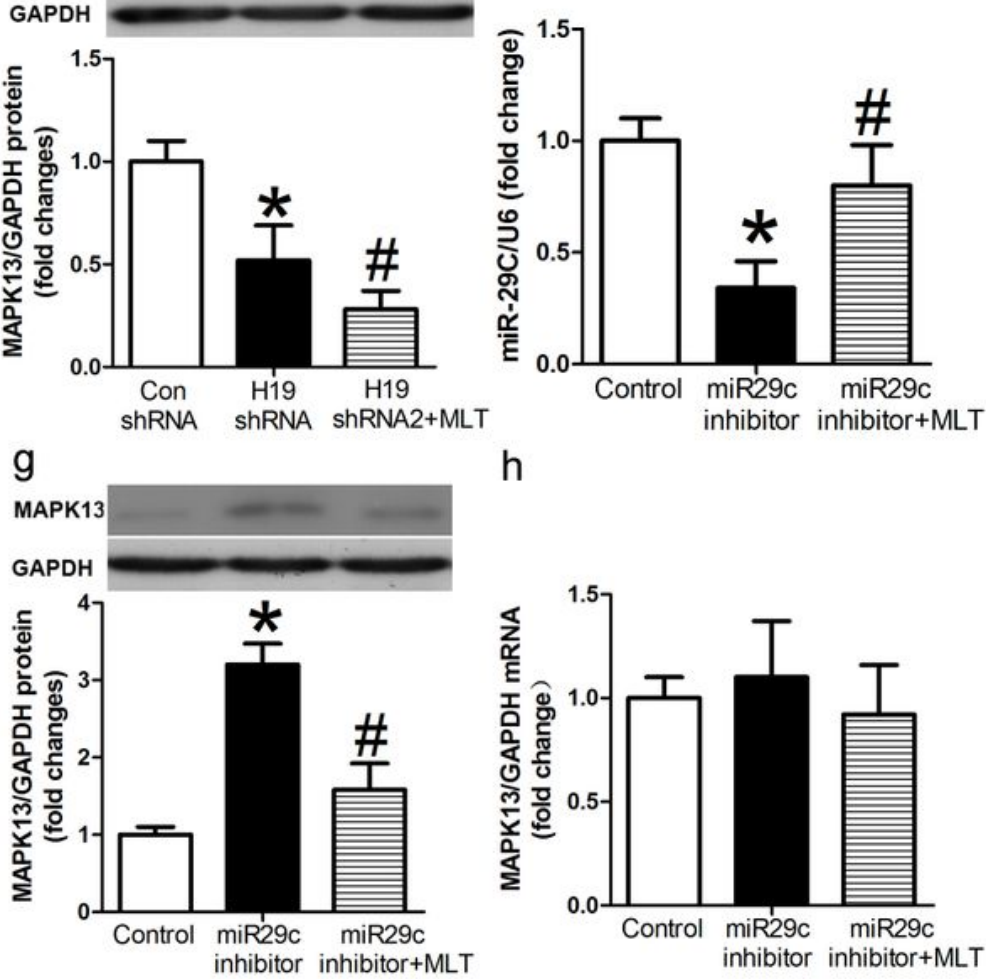

h

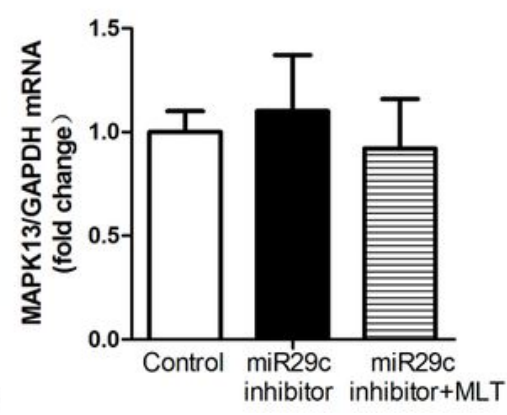

Figure 6

Melatonin affected the expressions of $\mathrm{H} 19$ and miR-29c in diabetic myocardium and regulated the networks of H19, miR-29c and MAPK13 in H9c2 cells. The higher expression of H19 (a) and lower expression of miR-29c (b) was abolished by melatonin treatment. The H19 (c), miR-29c (d) and MAPK13 
protein (e) changed significantly with H19-shRNA and melatonin treatments. Obvious expression changes of miR-29c (f) and MAPK13 protein (g) appeared in H9c2 cells with miR-29c inhibitor and melatonin treatments, but no change of MAPK13 mRNA(h). The data are presented as means \pm standard deviation. All experiments were performed at least in triplicate. ${ }^{*} \mathrm{P}<0.05$ vs. control group; $\# \mathrm{P}<0.05 \mathrm{vs}$. $\mathrm{DM}, \mathrm{H} 19-$ shRNA or miRNA-29c inhibitor group.

a
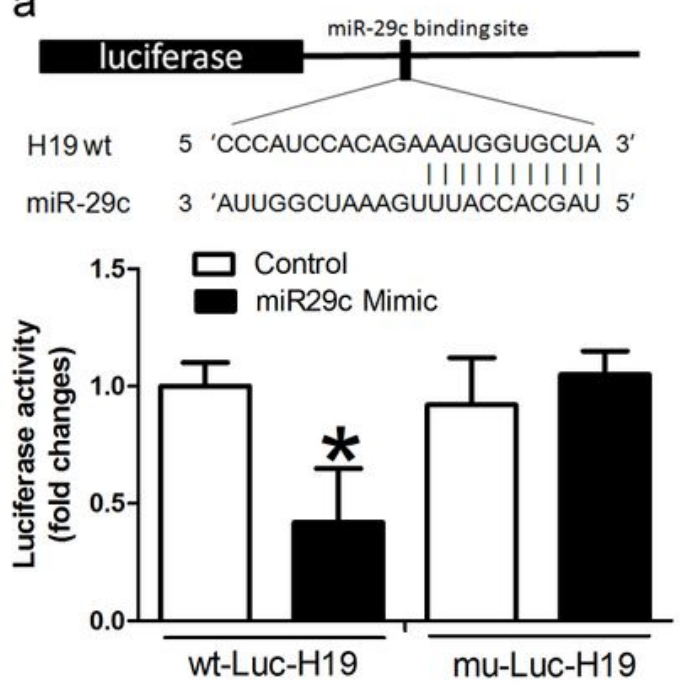

C

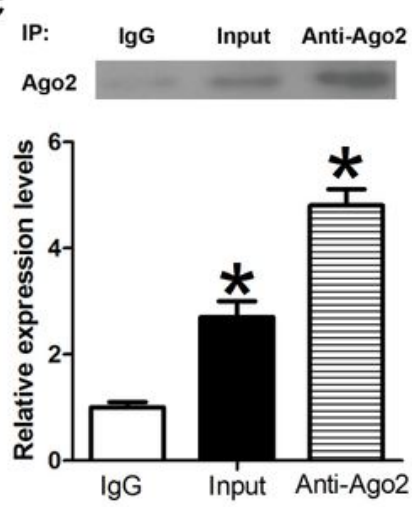

b

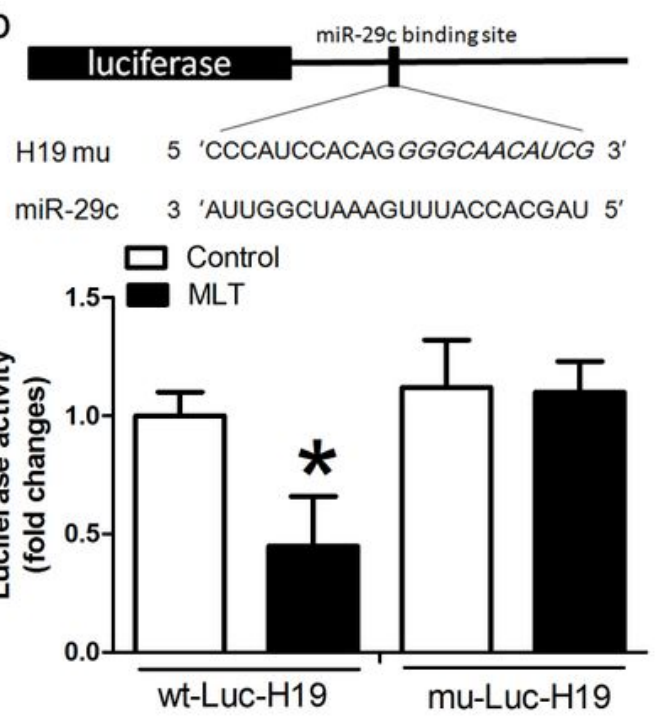

e f

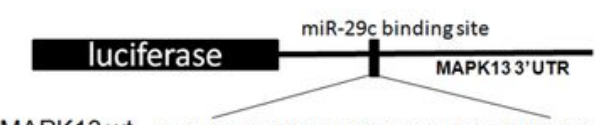

MAPK13 wt 5 'UGUCCCAGGGGCCUGUCGGUCAC 3' miR-29c 3 'CUUGUGGUCCUCUUU-AGCCAGU 5 '

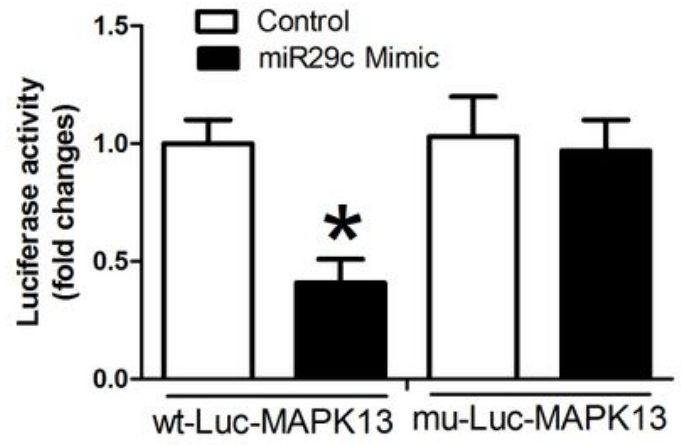

g

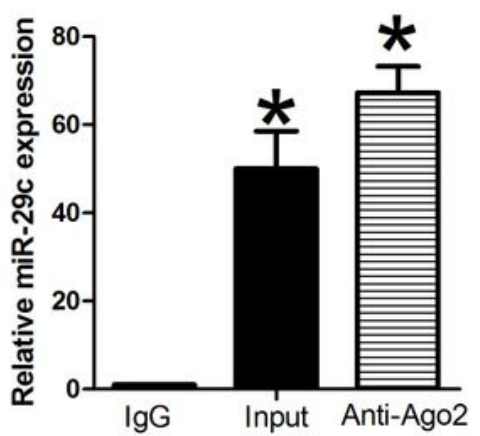

Figure 7 
The potential miR-29c binding sites in IncRNA H19 and MAPK13. (a)Wild type H19 3'-UTR binds with miR29c. MiR-29c mimic and luciferase constructs were co-transfected into H9c2 cells. Mutated H19 3'-UTR could not target with miR-29c mimic. (b) Melatonin combined with luciferase constructs were added to H9c2 cells. Cellular lysate from H9c2 was used for RNA immunoprecipitation with Ago2 antibody. Ago2 protein levels were measured by western blotting (c), and the expressions of $\mathrm{H} 19$ (d) and miR-29c (e) in the immunoprecipitate were measured by RT-PCR. The miR-29c 3'-UTR binding sites in wild type and mutated MAPK13 were shown $(f, g)$. MiR-29c mimic and melatonin were separately added to H9c2 cells with luciferase constructs. The data are presented as means \pm standard deviation. All experiments were performed at least in triplicate. ${ }^{*} \mathrm{P}<0.05$ vs. control group. 
a

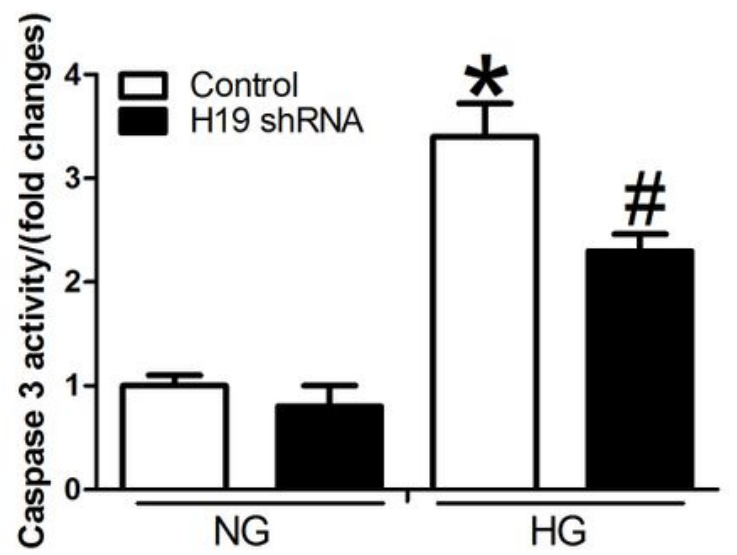

C

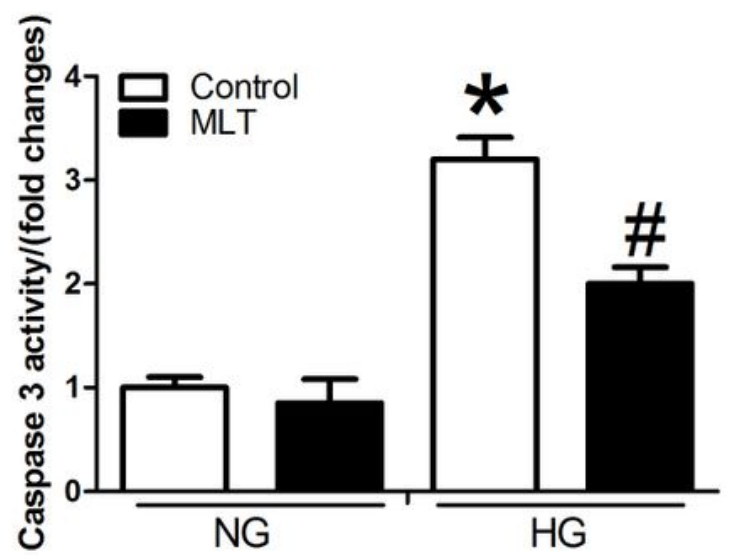

e

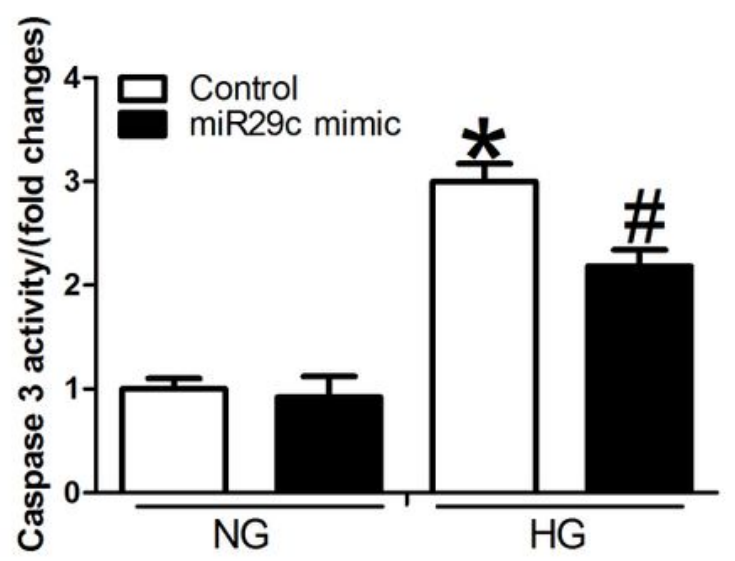

b

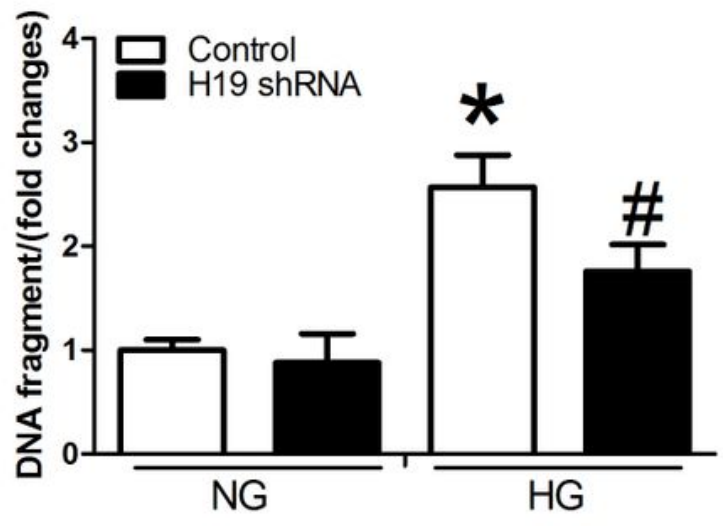

d

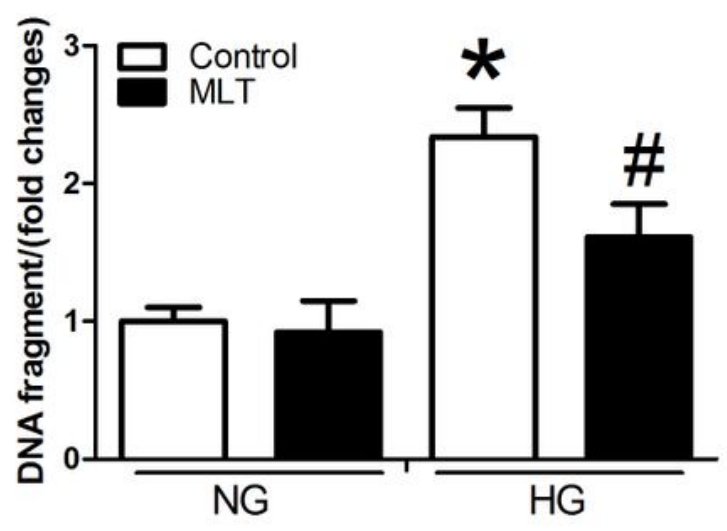

f

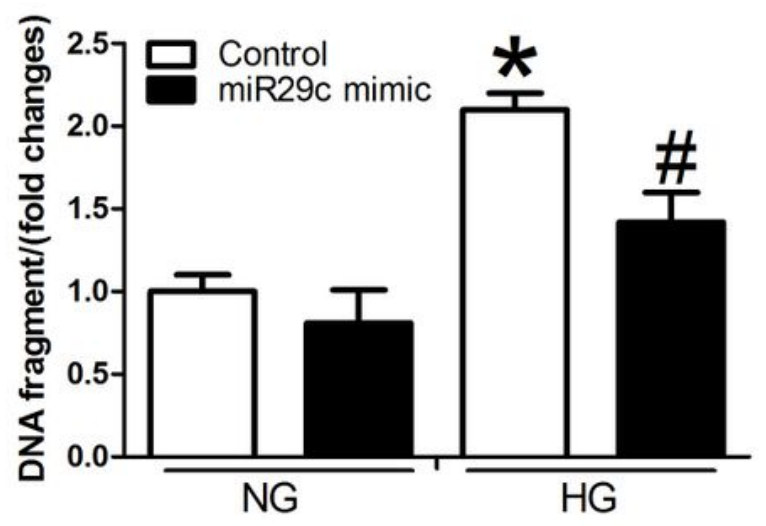

Figure 8

The effects of IncRNA H19 shRNA, melatonin and miR-29c mimic on apoptosis in H9c2 cells cultured in NG (NG: $5.5 \mathrm{mM}$ ) or HG (HG: $33 \mathrm{mM}$ ) medium. The decrease of caspase-3 activity (a, c,e) and DNA fragment $(b, d, f)$ indicated protective effects of above treatments on cells apoptosis in hyperglycemic condition. The data are presented as means \pm standard deviation. All experiments were performed at least in triplicate. ${ }^{*} \mathrm{P}<0.05$ vs. control group in NG. \#P $<0.05$ vs. control group in HG. 
a

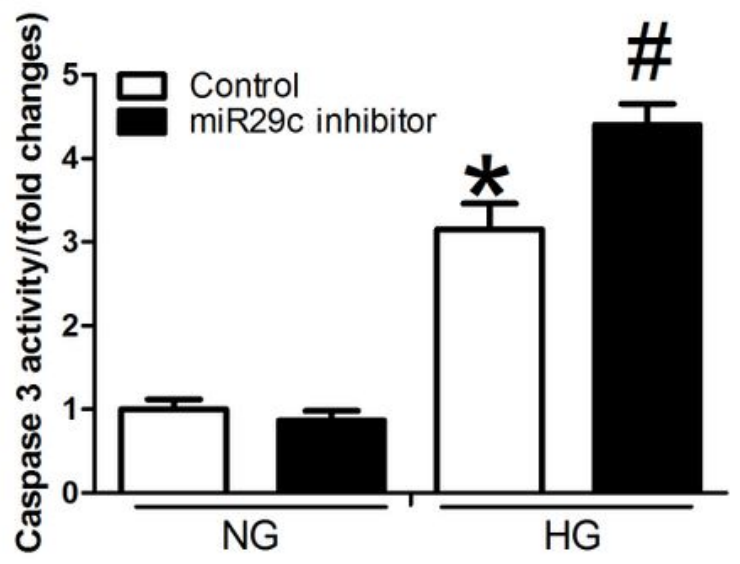

C

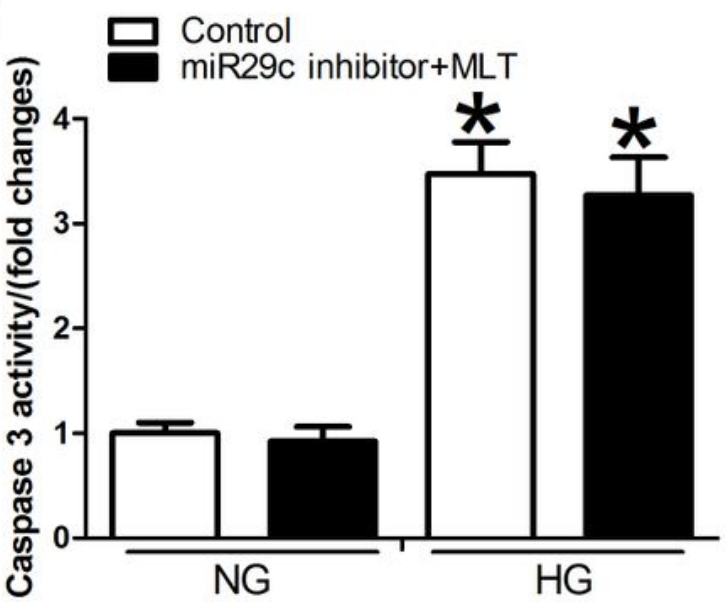

e

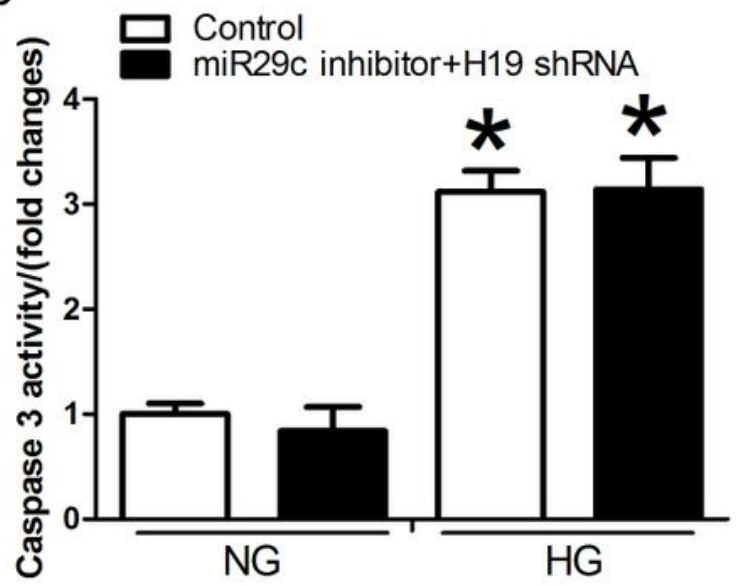

b

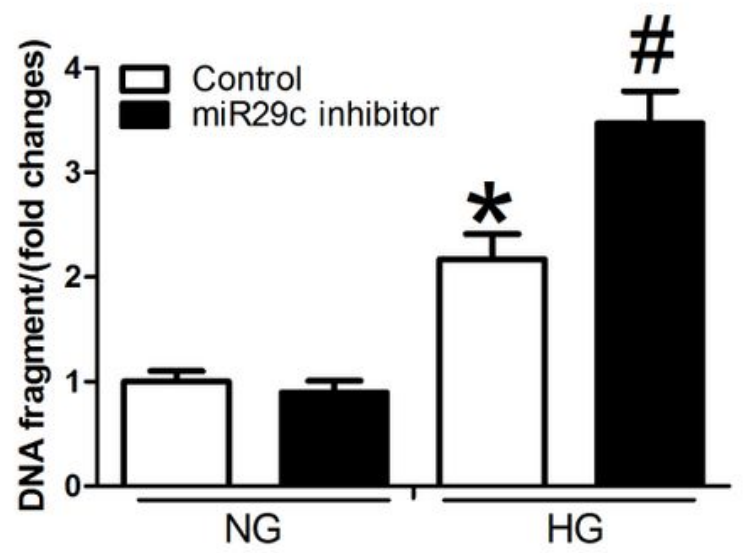

d

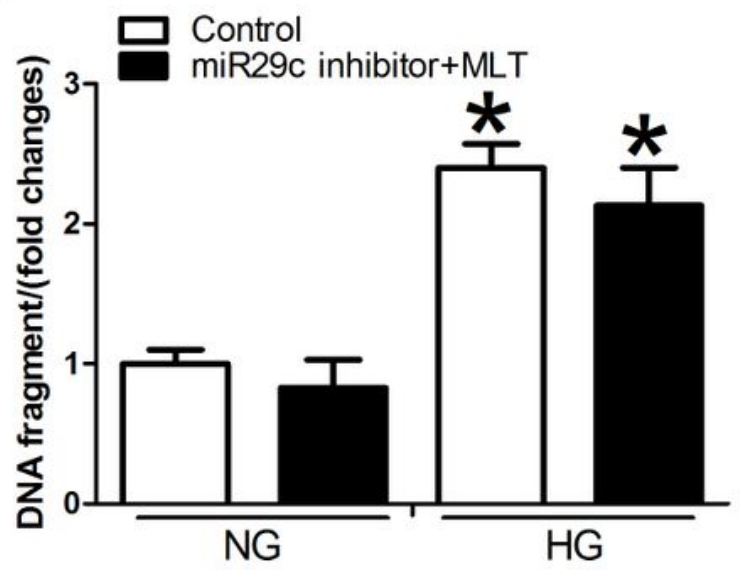

$f$

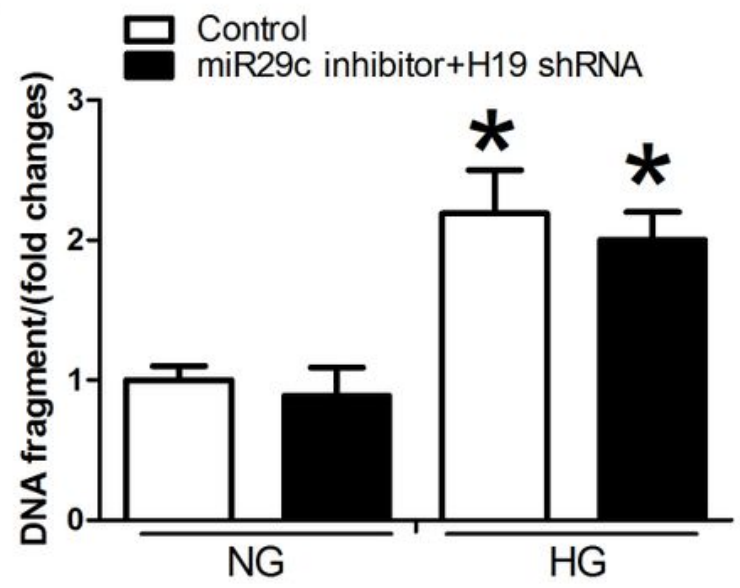

Figure 9

The effects of miR-29c inhibitor alone and in combination with H19-shRNA or melatonin on apoptosis of $\mathrm{H} 9 \mathrm{c} 2$ cells in hyperglycemic condition. $(\mathrm{a}, \mathrm{b})$ Data showed the pro-apoptotic effect of miR-29c inhibitor on H9c2 cells in hyperglycemic condition. (c-f) Melatonin and H19-shRNA reversed the pro-apoptotic effect of miR-29c inhibitor on $\mathrm{H} 9 \mathrm{c} 2$ cells in hyperglycemic condition. The data are presented as means \pm 
standard deviation. All experiments were performed at least in triplicate. ${ }^{*} \mathrm{P}<0.05$ vs. NG groups; $\# \mathrm{P}<$ 0.05 vs. control group in HG.

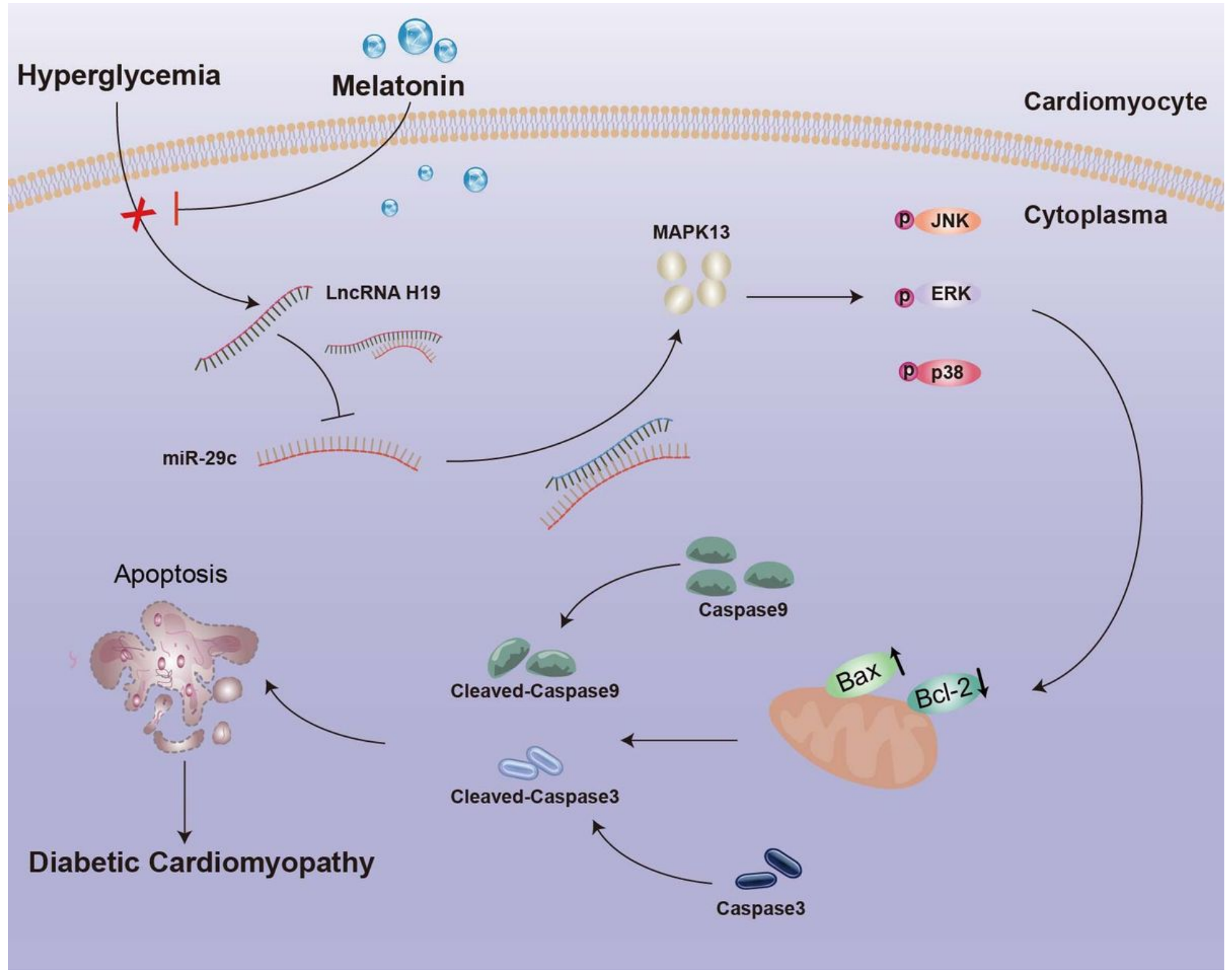

\section{Figure 10}

Schematic diagram illustrated that melatonin maintains ncRNAs homeostasis and reduces cardiomyocyte apoptosis in hyperglycemia condition via IncRNA H19/miR-29c/MAPK axis. 